\title{
The hippocampus and visual perception
}

\author{
Andy C. H. Lee ${ }^{1,3 *}$, Lok-Kin Yeung ${ }^{2}$ and Morgan D. Barense $e^{2,3 *}$ \\ 1 Department of Psychology (Scarborough), University of Toronto, Toronto, ON, Canada \\ 2 Department of Psychology (St. George), University of Toronto, Toronto, ON, Canada \\ ${ }^{3}$ Rotman Research Institute at Baycrest, Toronto, ON, Canada
}

\author{
Edited by: \\ Joel Voss, Northwestern University \\ Feinberg School of Medicine, USA

\section{Reviewed by:} \\ Joel Voss, Northwestern University \\ Feinberg School of Medicine, USA \\ Mark J. Buckley, Oxford \\ University, UK

\section{*Correspondence:} \\ Andy C. H. Lee, Department of \\ Psychology, University of Toronto \\ Scarborough, 1265 Military Trail, \\ Toronto, ON M1C 1A4, Canada. \\ e-mail: andylee@utsc.utoronto.ca \\ Morgan D. Barense, Department of \\ Psychology, University of Toronto, \\ 100 St. George Street, Toronto, ON \\ M5S 3G3, Canada. \\ e-mail: barense@psych.utoronto.ca
}

In this review, we will discuss the idea that the hippocampus may be involved in both memory and perception, contrary to theories that posit functional and neuroanatomical segregation of these processes. This suggestion is based on a number of recent neuropsychological and functional neuroimaging studies that have demonstrated that the hippocampus is involved in the visual discrimination of complex spatial scene stimuli. We argue that these findings cannot be explained by long-term memory or working memory processing or, in the case of patient findings, dysfunction beyond the medial temporal lobe (MTL). Instead, these studies point toward a role for the hippocampus in higher-order spatial perception. We suggest that the hippocampus processes complex conjunctions of spatial features, and that it may be more appropriate to consider the representations for which this structure is critical, rather than the cognitive processes that it mediates.

Keywords: hippocampus, perirhinal cortex, medial temporal lobe, memory, perception, amnesia, functional neuroimaging, neuropsychology

\section{INTRODUCTION}

Since the ground-breaking work of Scoville and Milner (1957), the hippocampus has been considered to be an integral component of a medial temporal lobe (MTL) memory system. A traditional view posits that the hippocampus forms an unitary memory system alongside other MTL structures, including the perirhinal cortex (PRC), entorhinal cortex, and parahippocampal cortex (Squire and Zola-Morgan, 1991; Squire et al., 2004; Squire and Wixted, 2011). According to this view, the MTL subserves long-term declarative memory exclusively (Cohen and Squire, 1980) and is crucial for the rapid acquisition of new information about facts and events. There are thought to be no clear functional differences between the various MTL structures and injury to any component of the MTL memory system is believed to produce a deficit in all forms of declarative memory proportional to the amount of damage to this system (Squire and Zola-Morgan, 1991; Squire et al., 2004; Squire and Wixted, 2011).

Alternative theories for the role of the MTL in memory argue that there is complete or relative functional segregation within the MTL, and that each structure is important for different mnemonic processes. One popular theory suggests that the hippocampus mediates recollective memory (i.e., remembering accompanied by the retrieval of contextual information) whereas the PRC is important for familiarity-based recognition (i.e., "feeling of knowing" in the absence of contextual retrieval) (Aggleton and Brown, 1999; Brown and Aggleton, 2001; Yonelinas et al., 2010). An alternative view argues that the hippocampus is critical for the rapid formation and retrieval of flexible associations between items such as individual percepts, context, and events, whereas the cortex surrounding the hippocampus, including the PRC, is essential for memory for individual items (Eichenbaum et al., 1992; Cohen and Eichenbaum, 1993; Moses and Ryan, 2006). More recently, a number of models related to these two theories have been suggested, including the Binding in Context model (BIC, Diana et al., 2007; Eichenbaum et al., 2007; Ranganath, 2010), and the Convergence, Recollection, and Familiarity Theory (CRAFT, Montaldi and Mayes, 2010). Both of these models consider recollection and familiarity to be a key factor to understanding functional segregation within the MTL, but critically, highlight the importance of considering the types of information that are being processed, with the hippocampus suggested to be important for processing associations between items and context, and the PRC involved in representing item information. Discussing the differences between these various views is beyond the scope of this review. The important point to make here is that in contrast to a unitary MTL memory view, all of these theories argue that differing patterns of MTL damage lead to distinct profiles of memory impairment, with hippocampal damage producing specific problems in mnemonic processing.

Against the backdrop of an abundant amount of data implicating the hippocampus and other MTL structures in declarative memory, there has recently been a steady accumulation of studies suggesting a role for the MTL in perception (e.g., Buckley and Gaffan, 2006; Bussey and Saksida, 2007; Murray et al., 2007; Graham et al., 2010). The majority of this work has focused on the PRC, and a putative role for this structure in the higherorder perception of objects, that is the processing of combinations of sensory features (e.g., within, but not limited to, the visual domain), which allow the successful sensation, identification, and discrimination of complex objects. More specifically, 
convergent research involving non-human primates (Eacott et al., 1994; Buckley and Gaffan, 1997, 1998; Buckley et al., 2001; Bussey et al., 2002, 2003), rodents (Bartko et al., 2007a,b), amnesic human patients (Barense et al., 2005, 2007, 2010b; Lee et al., 2005b,c, 2006, 2007; Lee and Rudebeck, 2010a) and computational modeling (Bussey and Saksida, 2002; Cowell et al., 2006, 2010b) has demonstrated that damage to the PRC results in a significant impairment in the discrimination of complex objects, which cannot be explained easily by a deficit in long-term declarative memory (see, however, Stark and Squire, 2000; Levy et al., 2005; Shrager et al., 2006; Clark et al., 2011; Kim et al., 2011). For instance, PRC damage has been shown to disrupt simultaneous and zero-delay matching-to-sample of perceptually similar object stimuli (Eacott et al., 1994), impair the discrimination of simultaneously presented objects that share a high number of visual features or objects that are presented from different viewpoints (Buckley et al., 2001; Bussey et al., 2002, 2003; Barense et al., 2005, 2007, 2010b; Lee et al., 2005b,c, 2006, 2007; Bartko et al., 2007a,b), and result in a deficit in the processing of structural coherency of single objects (Lee and Rudebeck, 2010a). Critically, these deficits exist in the context of intact perception of simple visual features such as color and size, as well as performance comparable to that of neurologically healthy control participants on standard neuropsychological tests of perception, highlighting the fact that the observed object perception deficits are only evident under particular circumstances. To consolidate the conclusions drawn from lesion studies, functional magnetic resonance imaging (fMRI) work in neurologically healthy participants has demonstrated significant PRC activity during the discrimination of complex, but not simple, objects (Devlin and Price, 2007; Lee et al., 2008; Barense et al., 2010a). Moreover, this PRC activity has been shown to be distinct from that associated with mnemonic processing (Lee et al., 2008; O’Neil et al., 2009, 2012; Barense et al., 2011a), highlighting the fact that the object perception deficits observed following PRC damage are unlikely to be a result of a primary deficit in long-term memory processing.

One theory that has been proposed to encapsulate the aforementioned PRC findings and articulate the functions of the PRC is the representational-hierarchical model (Murray and Bussey, 1999; Bussey et al., 2005; Murray et al., 2007; Saksida and Bussey, 2010). According to this view, the PRC is an extension of the representational hierarchy within the ventral visual stream (Ungerleider and Mishkin, 1982): rostral inferotemporal cortical regions, including PRC, form representations of complex conjunctions of stimulus features, whereas more caudal regions (e.g., $\mathrm{V} 4, \mathrm{TEO}$ ) represent the components from which these conjunctions are formed (Murray and Bussey, 1999; Riesenhuber and Poggio, 1999; Bussey and Saksida, 2002; Bussey et al., 2002). As such, the deficits that result from PRC damage can be best understood in terms of damage compromising high-level conjunctive representations of features (e.g., those comprising an object), leaving only lower-level representations (e.g., an object's shape or pattern) intact (Cowell et al., 2006). These complex conjunctive representations are critical when discrimination solely on the basis of simple features does not provide an easy solution to a problem (e.g., concurrent visual object discrimination).
For example, if visual features are rewarded when they are part of one object but not when they are part of another, or if two objects share many features in common (termed "feature ambiguity"), then these conjunctive representations are essential for successful performance. It is important to point out that while the representational-hierarchical model argues that the PRC processes complex conjunctions of features and, therefore, is critical to object perception, it does not claim that this structure does not have a role in memory. Rather, the PRC object representations are necessary for a variety of cognitive functions, and it is a disruption to these representations that leads to a deficit in both object perception and object memory (Saksida and Bussey, 2010; Cowell et al., 2010a).

Evidence for a critical role for the PRC in higher-order object perception has led to the possibility that the hippocampus-a structure heavily interconnected with the PRC, both directly and indirectly via the entorhinal cortex-may also be important for higher-order perceptual processing, for instance in the processing of combinations of spatial features, which allow the successful sensation, identification, and discrimination of complex spatial stimuli. Recent data indicating that the HC may be involved in the discrimination of complex scene stimuli support this idea (Lee et al., 2005b,c, 2006, 2007, 2008; Barense et al., 2010a). The notion that the hippocampus may be important for spatial cognition is not new. Forty years ago, O'Keefe and Dostrovsky (1971) discovered neurons in the rat hippocampus, which fired selectively when a rat was in a specific location. The discovery of these "place cells" pointed toward a role for the hippocampus in spatial navigation and cognition, and suggested that the hippocampus may subserve a cognitive map by containing a representation of our spatial environment (Tolman, 1948; O'Keefe and Nadel, 1978). A large number of studies, primarily in rodents, have since outlined a number of properties of these cells (O'Keefe et al., 1998; Best et al., 2001; Moser et al., 2008). The existence of cells that signal location (Ono et al., 1991, 1993; Hori et al., 2003) and spatial view information (Rolls et al., 1997; Robertson et al., 1998; Rolls, 1999) has also been demonstrated in nonhuman primates, and lesions to the hippocampus in monkeys have been shown to impair spatial location memory (Murray et al., 1998; Hampton et al., 2004; Lavenex et al., 2006). In addition to this, there has been substantial converging evidence from studies in humans, with electrophysiological and fMRI research reporting the presence of place cells or place cell like activity in the human hippocampus (Ekstrom et al., 2003; Hassabis et al., 2009), neuropsychological accounts of spatial memory impairments following hippocampal damage (e.g., Bohbot et al., 1998; Holdstock et al., 2000; Rosenbaum et al., 2000, 2005; Spiers et al., 2001; Burgess et al., 2002; King et al., 2002; Feigenbaum and Morris, 2004; Maguire et al., 2006; Taylor et al., 2007; Bird et al., 2008), and MRI studies highlighting the involvement of the human hippocampus in spatial navigation (Maguire et al., 1997, 1998; Spiers and Maguire, 2006, 2007; Igloi et al., 2010; Morgan et al., 2011).

Despite an overwhelming amount of data supporting a role for the hippocampus in spatial cognition, it is fair to say that the vast majority of this work has been in the context of spatial memory tasks, or paradigms in which the learning and recall of spatial 
information is a vital component (e.g., spatial navigation). The suggestion that the hippocampus is critical for higher-order spatial perception goes beyond the mnemonic domain and argues that the hippocampus is critical for complex spatial processing irrespective of whether mnemonic processing is required (Gaffan, 2001, 2002). In this review, therefore, we will first present evidence from a number of experiments in humans that suggest a role for the hippocampus in complex spatial perception. We will focus on studies that have used experimental paradigms that do not possess an explicit long-term memory component and, therefore, speak directly to the idea that the hippocampus may be important for processes beyond declarative memory. We will then consider human studies that have failed to find hippocampal involvement in similar spatial perception tasks and attempt to reconcile these disparate findings. Following this, we will discuss how the hippocampus may contribute to complex spatial perception, taking into account studies in humans, non-human primates, and rodents that have involved "perceptual" or mnemonic tasks. In particular, we will consider how a role in complex spatial perception relates to other hippocampal functions/processes such as allocentric spatial processing, pattern completion and separation, and mnemonic processing. Finally, we will propose future directions of research that could facilitate our understanding of the perceptual processes for which the hippocampus may be essential.

\section{DIRECT EVIDENCE FOR A ROLE FOR THE HIPPOCAMPUS IN COMPLEX SPATIAL PERCEPTION}

To investigate the role of the hippocampus in spatial perception, Lee and colleagues performed a series of experiments designed to tax the perception of complex scene stimuli (i.e., scenes that could not be discriminated easily on the basis of a single feature) in the absence of explicit long-term memory demands. In the first study, amnesic patients with MTL damage were tested on complex visual discriminations involving pairs of images that had been morphed together to varying degrees such that they shared between 0 and $49 \%$ of features (Lee et al., 2005c). There were five image categories in total, with each category consisting of 2 or more pairs of exemplars (each pair administered in separate trial blocks). The five categories were: faces (2 pairs of famous faces and 2 pairs of non-famous faces), objects (1 pair of non-living items and 1 pair of animals), scenes (3 pairs of outdoor scenes), abstract art (3 pairs) and colors (3 pairs). On each trial, participants distinguished which of two stimuli was most similar to a third stimulus, the latter being one of the original exemplar images used to create the morphed stimuli. All stimuli in a given trial were presented concurrently (Figure 1). The target and foil stimuli on each trial were unique, although the same two exemplar images were used to create all the trials for each stimulus block. Patients with selective hippocampal damage performed normally when discriminating face, object, abstract art, and color stimuli, but were significantly impaired when discriminating images of real world scenes. Importantly, the hippocampal patients made an increased number of errors as the percentage of shared features across the scenes in each trial increased (i.e., the target and foil stimuli were more similar to each other due to a greater degree of morphing between the two exemplar images). Patients with broader MTL damage

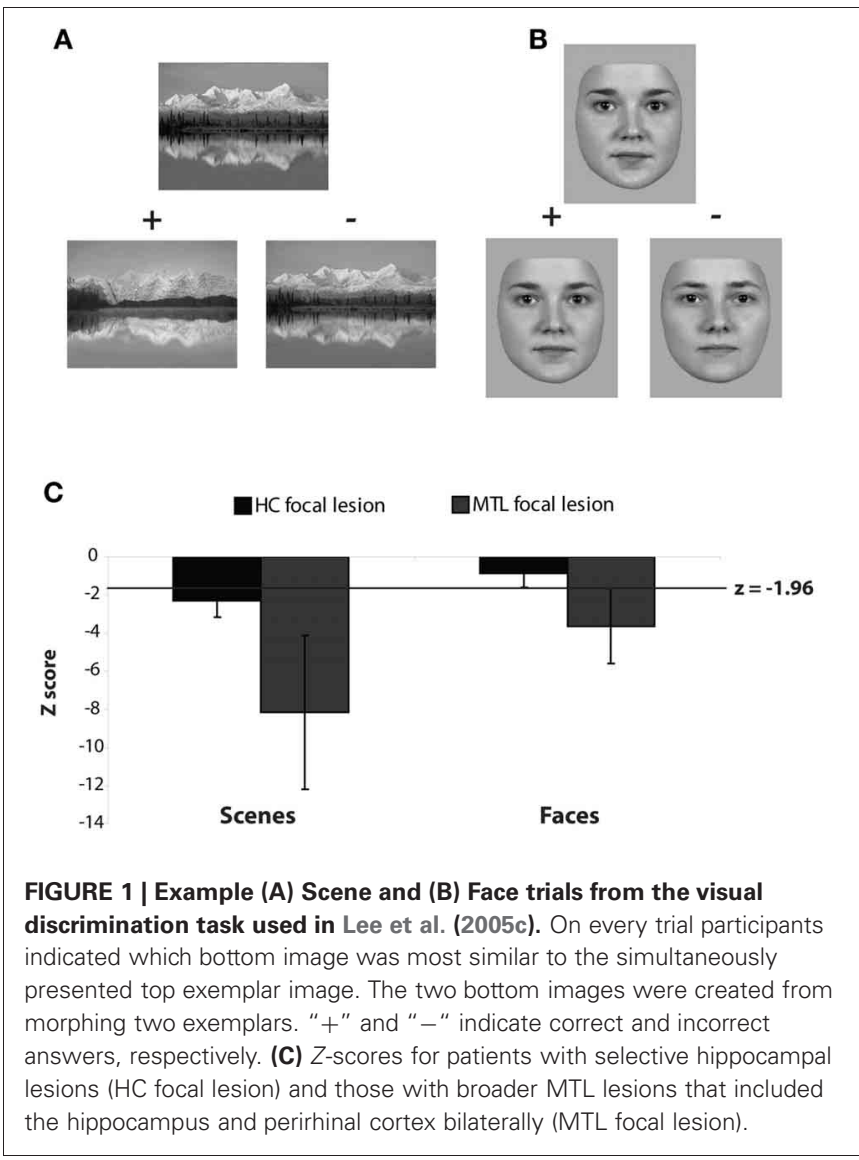

that included the hippocampus and perirhinal cortex also demonstrated an impairment in discriminating scenes but, critically, also showed impairments in discriminating faces, and (to a lesser extent) objects (Figure 1). Because this experimental paradigm has a minimal mnemonic demand (the target and choice stimuli are presented simultaneously, and there is no requirement to remember information across trials), these findings indicate that the hippocampus is critical for discriminating scenes with a high degree of overlap, whereas the PRC is responsible for discriminating faces and objects that share a large proportion of features.

An alternative paradigm that has been used to assess the involvement of the hippocampus in spatial perception is the oddity judgment task. In one such study, participants were asked to select the odd-one-out from an array of four simultaneously presented stimuli (Lee et al., 2005b). The stimuli were either images of 3D virtual reality indoor rooms (see Figure 2) or photographs of unfamiliar faces, and the stimuli on each trial were presented either from the same viewpoint ("same view" condition) or different viewpoints ("different view" condition). For instance, in the same view scene condition, three identical images of the same room are presented with a fourth image of a different room. In contrast, in the different view scene condition three different viewpoints of the same room are shown alongside another viewpoint of a different room. In both the same and different view scene conditions, the target scene on each trial is 

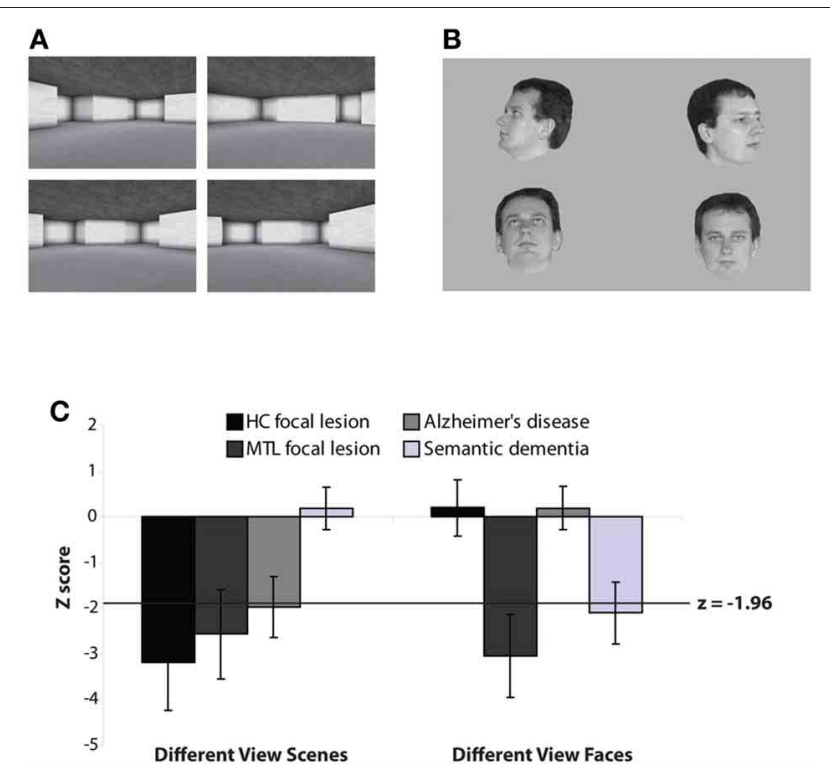

FIGURE 2 | Example trials from the (A) Different View Scenes and (B) Different View Faces oddity judgment tasks used in Lee et al. (2005b, 2006). On each trial participants indicated which image was different from the other three, simultaneously presented, images. All stimuli were trial-unique. For both examples, the odd-one-out is in the top right corner. (C) Z-scores for patients with selective hippocampal lesions ( $\mathrm{HC}$ focal lesion), broader MTL lesions that included the hippocampus and perirhinal cortex bilaterally (MTL focal lesion), cases with Alzheimer's disease (predominant $\mathrm{HC}$ damage), and patients with semantic dementia (predominant PRC damage).

similar to the three foils but differs with respect to the size or location of one or more features such as a wall, room cavity or window. Notably, successful performance on the different view scene condition is dependent upon the ability of participants to process multiple spatial relationships between individual features in the scene, whereas the same view scene task places a reduced demand on this process. No stimuli are repeated across trials in any condition and because the participants are making perceptual judgments on simultaneously presented stimuli, no explicit longterm memory is necessary for successful performance. In support of a role for the hippocampus in complex spatial perception, cases with selective hippocampal lesions were found to be severely impaired when the scenes were shown from different viewpoints, but not when the scenes were shown from the same viewpoint. Critically, these patients performed normally when the stimuli to be discriminated were faces, regardless of viewpoint manipulation (Figure 2). In contrast, patients with both hippocampal and PRC damage demonstrated significant deficits in both scenes and faces shown from different views, but performed normally with scenes and faces shown from the same view. In a followup study, the same task was administered to groups of patients with Alzheimer's disease (AD) and patients with semantic dementia (SD) (Lee et al., 2006). At least in the early stages of disease progression, $\mathrm{AD}$ and $\mathrm{SD}$ are associated with relatively greater hippocampal and PRC atrophy, respectively (Davies et al., 2004), and thus, provide another patient population for the investigation of the effects of human MTL damage. To underline previous findings in focal lesion cases, it was found that $\mathrm{AD}$ patients were impaired compared to healthy controls in both viewpoint conditions when scenes were used as the stimuli, but not when faces were employed. In a striking double dissociation, SD patients showed intact performance for both scene conditions and the face same view task, but were impaired when asked to discriminate faces shown from different viewpoints (Figure 2). The AD group impairment on same view scene oddity judgment was not predicted given the intact performance of the selective hippocampal lesion patients on this exact condition in Lee et al. (2005b). It is interesting to note, however, that the range of performance on this condition was similar across the AD and hippocampallesioned patient groups, despite the hippocampal lesion cases not being significantly different from their control group. It is conceivable, therefore, that the same view scene oddity judgment task is not an entirely hippocampal-independent task, and does place some demand (albeit relatively reduced compared to the different view scene task) on the spatial perceptual processes for which the hippocampus may be critical.

To rule out the possibility of dysfunction to regions beyond the hippocampus underlying the aforementioned findings, convergent evidence from fMRI research in neurologically healthy participants is crucial. Lee et al. (2008) scanned healthy participants while they performed a modified version of the oddity judgment task from Lee et al. (2005b). In this version, participants completed blocks of trials of different view face and scene oddity discriminations, as well as a control size oddity judgment condition, in which three squares of the same size were presented with a fourth square of a different size on each trial. When compared to the size task, the face oddity judgment task was associated with activation in the left PRC, and bilaterally in the anterior and posterior hippocampus, whereas the scene oddity judgment task led to bilateral activation in the posterior hippocampus and parahippocampal cortex. Importantly, a direct contrast between the face and scene oddity conditions revealed that the former was associated with significantly greater activity in the left PRC and bilateral anterior hippocampus, highlighting the relative importance of the PRC to object but not scene perception. On the other hand, significantly greater activity was seen in the posterior hippocampus and parahippocampal cortex bilaterally during the scene oddity condition.

To examine the effect of stimulus viewpoint, an ensuing study used fMRI to investigate MTL activity in neurologically healthy participants during a series of oddity judgments involving faces, scenes, or novel objects that were presented from either identical or different viewpoints (Barense et al., 2010a). Consistent with studies in patients demonstrating the critical importance of viewpoint (Lee et al., 2005b, 2006; Barense et al., 2007), increased perirhinal activity was observed when participants distinguished between faces and objects presented from different, compared to identical, viewpoints. The posterior hippocampus, by contrast, showed an effect of viewpoint for scenes as well as faces. An effect of viewpoint in the posterior hippocampus for faces was not predicted given the ability of patients with hippocampal atrophy to make successful different view face oddity judgments (Lee et al., 2005b, 2006), and was, at the time of the study, interpreted as a 
reflection of the recruitment of match-mismatch processes that may be subserved by the hippocampus (Kumaran and Maguire, 2006b, 2007). These processes, which are not specific to any stimulus category, may be involved in the detection of items that are matching or non-matching on an oddity trial, but are not critical to successful performance as demonstrated by the intact performance of patients with hippocampal damage on face as well as object oddity judgment (Lee et al., 2005b, 2006; Barense et al., 2007).

In summary, a series of convergent neuropsychological and functional MRI studies across different patient groups (i.e., focal lesion amnesics and cases with neurodegenerative disease) and neurologically healthy participants have suggested that the hippocampus is involved during complex spatial discrimination problems. The fact that these observations arose from experimental paradigms in which there is no explicit long-term memory demand underlines the notion that the MTL is critical for processes beyond long-term declarative memory, and suggests that the hippocampus is important for higher-order spatial perception. This is consistent with the well-established role of the hippocampus in spatial cognition, but crucially, extends its involvement in spatial processing beyond memory and the encoding and retrieval of spatial representations.

\section{DOUBTS SURROUNDING A ROLE FOR THE HIPPOCAMPUS IN COMPLEX SPATIAL PERCEPTION}

Given the traditional belief that the hippocampus is a central component of an exclusive long-term declarative memory system (Squire et al., 2004; Squire and Wixted, 2011), and the fact that patients with MTL lesions typically perform within the normal range on standard neuropsychological assessments of visual perception (for a review see Lee et al., 2005a), it is not surprising that the idea that the hippocampus may also be important for spatial perception has come under considerable scrutiny (Baxter, 2009; Suzuki and Baxter, 2009; Suzuki, 2009). We will discuss some of the main criticisms leveled at this idea below.

\section{THE HIPPOCAMPUS AND SPATIAL DISCRIMINATION DEFICITS: PERCEPTION OR LEARNING?}

One criticism that has often been raised by proponents of a MTL memory system is that neuropsychological findings purporting to support a role for the hippocampus in spatial perception can be explained by a primary impairment in learning in the patients with MTL damage. The basis of this claim stems largely from the design of the visual discrimination paradigm that was used in Lee et al. (2005c). In this study, although each trial was unique, the same pair of exemplars was repeatedly blended to varying degrees to create the target and foil items in each block of trials. It is plausible, therefore, that the matched controls were able to benefit from learning across trials, leading to a significant performance improvement compared to memory-impaired MTL lesion patients who could not benefit from this learning. Evidence to support this explanation comes from two recent studies claiming that the visual discrimination impairments described by Lee et al. (2005c) disappear when healthy controls are prevented from learning across trials (Shrager et al., 2006; Kim et al., 2011). In these studies, a similar task to that used by Lee et al. (2005c) was administered to patients with selective hippocampal lesions (Shrager et al., 2006; Kim et al., 2011) and patients with extensive MTL damage (Shrager et al., 2006). In these tasks, participants were instructed to indicate which of two images (created by morphing two exemplar images) was most similar to a third simultaneously presented image. Critically, however, two versions of this task were administered: one in which the target and foil stimuli on each trial were created by the same pair of exemplars ("repeated" condition, as in Lee et al., 2005c); and another in which the target and foil stimuli on each trial were created by different pairs of exemplars ("trial-unique" version). Focusing on the findings for scene stimuli only, Shrager et al. (2006) found that both hippocampal and extensive MTL lesion patients were not impaired in the discrimination of real-world scenes either when the exemplar pair was repeated or when trial-unique exemplar pairs were used across trials. On the other hand, Kim et al. (2011) found that the hippocampal patients were not impaired when trial-unique exemplar pairs of virtual reality 3D rooms were used to create the target and foil stimuli, but did exhibit a significant deficit when the same exemplar pair was used repeatedly.

At first glance, the two aforementioned studies appear to suggest that the spatial discrimination deficits demonstrated by Lee et al. (2005c) can be explained by learning in controls and that the hippocampus, therefore, does not subserve higher-order spatial perception. On further consideration, however, such an explanation seems unlikely for multiple reasons. First, if learning in controls is believed to take place when the same exemplar pair is repeated across trials, it is not clear why this did not occur in Shrager et al. (2006), in which a patient deficit was not observed for scene discriminations, even though these stimuli had also been repeated. No explanation has been offered for this discrepancy by proponents of an exclusive MTL memory system and, from the perspective of long-term learning facilitating concurrent visual discrimination, there is no systematic reason why control participant learning would occur in one study (Kim et al., 2011) but not another (Shrager et al., 2006). It is possible that the stimuli in the former (virtual reality scenes) were more memorable to the healthy participants than those in the latter (real world scenes), although there is no obvious reason why this would be the case. Second, the data from Kim et al. (2011) appear to indicate that there was a difference in performance between patients and controls even early on in the trial-unique task (i.e., first and second blocks of trials), with no obvious increment in this difference as the task progressed. This pattern of findings undermines the notion of control learning across trials, which one would predict to occur gradually over a number of trials and lead to a significant increase in the difference between patient and control discrimination performance over time. Third, it is important to highlight that Lee et al. (2005c) specifically plotted performance in controls and patients as a function of time (i.e., accuracy across blocks of trials) and found that neither patients nor controls improved their performance as a result of learning over trials. In fact, the control participants demonstrated a significant decrease in performance over time, suggesting that memory for early trials had a negative, not positive, impact on their discrimination ability in later trials. Finally, a learning-based explanation is unable to account for studies reporting that damage to the hippocampus 
impaired spatial oddity judgment of trial-unique stimuli (Lee et al., 2005b, 2006). There are no repeating stimuli in this spatial oddity judgment task (i.e., each stimulus is novel) and unlike the discrimination task used by Lee et al. (2005c), the foils and targets are not created by blending exemplar stimuli.

Proponents of an exclusive MTL memory system have argued that in perceptual tasks in which stimuli are truly trial-unique (i.e., in the oddity judgment paradigms referred to above), the ability of controls, and not amnesic patients, to rapidly encode individual items into long-term memory may facilitate the discrimination of simultaneously presented stimuli (Kim et al., 2011). We now have clear evidence that this is unlikely to be the case. In a recent study (Lee et al., in preparation), neurologically healthy participants were scanned using fMRI while they carried out a large number of different view scene oddity judgment trials. Crucially, the participants were given a surprise recognition memory test after scanning, in which the trials seen during scanning were intermixed with trials that had not been presented previously. Thus, each trial could be classified into one of four categories: (1) correct oddity and subsequently recognized; (2) correct oddity and subsequently forgotten; (3) incorrect oddity and subsequently recognized; and (4) incorrect oddity and subsequently forgotten. Across both univariate and multivariate analysis techniques, it was found that correct oddity judgment trials were associated with significantly greater hippocampal activity than incorrect oddity judgment trials. Importantly, this difference was present irrespective of whether a trial was subsequently recognized or forgotten, undermining the idea that encoding into long-term memory is necessary (or even beneficial) for successful spatial oddity judgment.

If incremental learning and long-term memory encoding in controls can be accounted for, it is not immediately clear how discrepant findings concerning the impact of hippocampal lesions on concurrent discrimination of spatial stimuli can be reconciled. One possibility, favored by proponents of an exclusive MTL memory system, is that variations in the size and extent of patients' lesions can explain the presence or absence of spatial discrimination impairments following hippocampal damage (see next section for a discussion of this issue). Our preferred explanation is that the types of spatial stimuli that are presented are critical to eliciting spatial discrimination deficits in hippocampal lesion patients. More specifically, discriminations that involve scene stimuli that cannot be discriminated easily on the basis of single spatial features but necessitate the processing of multiple spatial features and relationships are likely to be disrupted as a result of injury to the hippocampus. By contrast, scene stimuli that can be discriminated using a single-feature matching strategy would not require the hippocampus. Although the morphing of scenes has been used to create the stimuli in the aforementioned contradictory studies (Lee et al., 2005c; Shrager et al., 2006; Kim et al., 2011), it is important to note that even high-level morphing does not necessarily eradicate the presence of single features that can facilitate discrimination, perhaps explaining discrepant findings between studies that have used this approach (Baxter, 2009) (Figure 3). To a certain extent, the different view scene oddity judgment task does not suffer from the same problem (Lee et al., 2005b, 2006). In this paradigm, the four scenes shown on

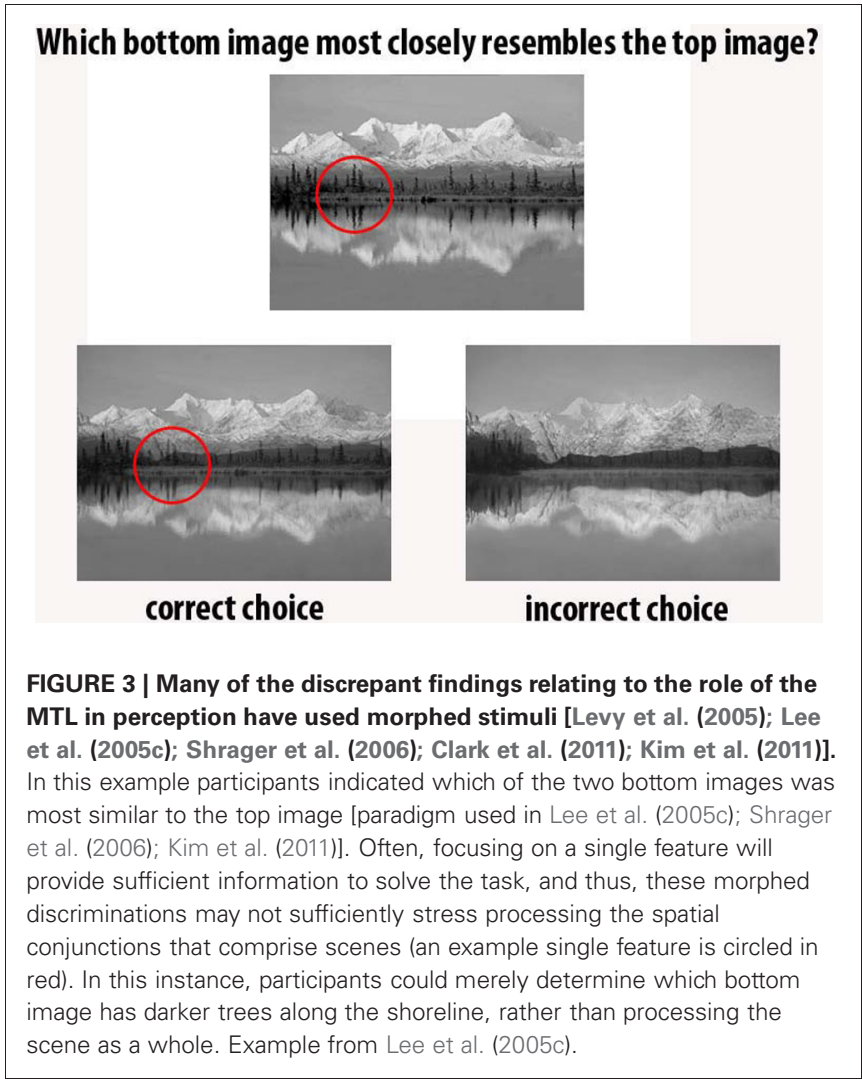

each trial are distinct from one another due to the use of different viewpoints, and thus, participants cannot solve the discrimination by simply focusing on a single feature and examining how this feature varies across the four scenes.

\section{THE HIPPOCAMPUS AND SPATIAL DISCRIMINATION DEFICITS: DAMAGE BEYOND THE MTL?}

One repeated criticism is that the patients who have been reported to possess visual discrimination deficits may possess unreported damage to cortical regions beyond the MTL that are more typically associated with visual perceptual processes (Suzuki, 2009; Kim et al., 2011), including posterior fusiform and temporal cortical regions, and extrastriate visual areas such as the parahippocampal place area (PPA) (Epstein and Kanwisher, 1998), lateral occipital complex (LOC) (Malach et al., 1995; Kourtzi and Kanwisher, 2000), and fusiform face area (FFA) (Kanwisher et al., 1997; McCarthy et al., 1997), which have been implicated in the perception of scenes, objects, and faces, respectively. In the neuropsychological studies that first suggested a role for the MTL in perception (Barense et al., 2005; Lee et al., 2005b,c), electronic structural MRI scans were not available for the patients that were assessed. Consequently, brain damage was evaluated via the qualitative visual assessment of a number of key regions on a select number of scan slices, using a visual rating scale that has been previously validated against volumetric measures (Galton et al., 2001). Although this approach indicated that only two out of four hippocampal lesion patients had significant damage beyond the hippocampus to the parahippocampal gyrus (patients 
HC1 and HC3), and one of three patients with extensive MTL damage (patient MTL1) possessed significant damage to lateral temporal cortex (Barense et al., 2005), it is possible that that additional damage in these and other patients went undetected in scan slices that were not visually rated. To address this issue, we have now, where possible, re-acquired MRI scans in the same patients and conducted detailed volumetric analyses. These analyses have revealed that structural damage to regions beyond the MTL are unlikely to account for all of the visual discrimination deficits that have been observed (Lee et al., 2008; Lee and Rudebeck, 2010a; Barense et al., 2011b). For instance, in a detailed description of two of these cases (HC3 and MTL3), Lee and Rudebeck (2010a) reported that neither patient had significant damage to the posterior lateral fusiform gyrus or posterior lateral temporal cortex. Moreover, contrary to the findings of previous qualitative visual ratings, patient $\mathrm{HC} 3$ did not possess significant atrophy to the parahippocampal cortex. Her damage was entirely confined to the hippocampus, suggesting that the deficit in spatial oddity judgment cannot be easily explained by structural damage beyond the hippocampus. More recently, detailed volumetric analyses of another hippocampal lesion patient's structural MRI scan (patient HC1) have also been reported, indicating no significant damage beyond the hippocampus (Barense et al., 2011b). Given that patients $\mathrm{HC} 1$ and HC3 are both significantly impaired on scene oddity judgment but have no significant atrophy to regions outside of the hippocampus, we feel confident in concluding that an intact hippocampus is critical for tasks of complex spatial discrimination.

It is important, however, to acknowledge that the failure to find obvious structural damage to gray matter in regions beyond a lesion site does not suggest that any observed cognitive deficits can unequivocally be attributed to the lesion (e.g., Mumby et al., 1996). For example, it is possible that the perceptual deficits observed in patients with MTL damage can be accounted for by functional impairment in structurally intact regions beyond the MTL, as well as degeneration in white matter tracts that can only be detected using techniques such as diffusion tensor imaging (DTI). In response to this uncertainty, it is worth making three points. Firstly, according to our knowledge, the vast majority of neuropsychological studies that have made claims about the functions of MTL structures on the basis of impairments in patients with MTL damage have not carried out detailed structural MRI, functional MRI, as well as DTI in the patients. Thus, any doubts concerning functional and white matter integrity in MTL studies of visual discrimination are equally applicable to MTL studies of mnemonic processing. Secondly, Lee and Rudebeck (2010a) carried out fMRI on patients HC3 and MTL3 during the presentation of scene, object, and face stimuli (i.e., a standard functional localizer task). Not only were functionally defined extrastriate areas (i.e., PPA, FFA, LOC) evident in both of these cases, but the pattern of activity in these regions was not significantly different to that in matched controls other than in the LOC in patient HC3, which demonstrated relatively greater activity in response to scenes and objects. Finally, we have recently used DTI and AMRI to examine the integrity of white matter tracts and resting state networks in patients HC3 and MTL3 and found that there were no obvious differences between patients and matched controls in the integrity of white matter tracts outside of the MTL, or differences in resting state networks, which can account entirely for the patients' visual discrimination deficits (Rudebeck et al., in preparation). Thus, these preliminary analyses further underscore our belief that the visual problems these patients exhibit cannot be attributed to dysfunction beyond the MTL.

\section{THE HIPPOCAMPUS AND SPATIAL DISCRIMINATION DEFICITS: PERCEPTION OR WORKING MEMORY?}

An alternative explanation for the spatial discrimination deficits seen in patients with hippocampal damage is that they reflect an impairment in short-term spatial working memory, rather than higher-order spatial perception. Over the past 10 years, there has been a steady accumulation of findings implicating the MTL in working memory processes. For instance, the hippocampus and surrounding MTL structures have been suggested to be involved in the short-term maintenance of complex (e.g., images of faces and scenes) and simple (e.g., shapes) visual stimuli, and relational information (e.g., spatial relationships between individual items) (Ranganath and D'Esposito, 2001; Stern et al., 2001; Ryan and Cohen, 2004; Hannula et al., 2006; Nichols et al., 2006; Olson et al., 2006a,b; Hartley et al., 2007; Hannula and Ranganath, 2008; Cashdollar et al., 2009; Bird et al., 2010; Warren et al., 2010) (see however, Shrager et al., 2008; Jeneson et al., 2011). Because studies that have reported visual discrimination deficits after MTL damage have all relied on experimental paradigms that require participants to compare across multiple simultaneously presented images, it is plausible that poor performance may reflect a deficit in MTL-mediated working memory processes, and, therefore, an inability to maintain and compare multiple stimuli in working memory. Supporting evidence for this view comes from studies that showed an absence or inconsistent patterns of impairment when patients with MTL damage were administered visual discrimination tasks involving simultaneously presented stimuli, but robust differences compared to control participants when a short delay was implemented between the presentation of the target and choice stimuli, thereby increasing working memory demand (Hartley et al., 2007; Cashdollar et al., 2009; Bird et al., 2010; Warren et al., 2010). For example, Cashdollar et al. (2009) found that patients with hippocampal atrophy were significantly impaired on a delayed-match-to-sample task that required the participants to maintain spatial relational information over a short period. In this task, a sample scene image was presented briefly and, after a $5 \mathrm{~s}$ delay, the patients were required to select the matching image from a pair of choices, with the foil differing from the target by the addition of a new object or the spatial shifting or deletion of an existing object. Whereas the patients struggled on this task, no deficit was reported when the need to maintain spatial relational information in working memory was removed (for instance when the two choice stimuli were unrelated to the sample image and the participants were asked to determine whether these two stimuli were identical). A similar effect of working memory maintenance was also observed by Hartley et al. (2007) and Bird et al. (2010), who administered a topographical perception and working memory test to patients with focal hippocampal damage, or hippocampal 
atrophy as a result of $\mathrm{AD}$ or amnestic mild cognitive impairment (a-MCI). Participants were presented with an image of a 3D virtual reality landscape and were required to select a matching image from an array of choices presented simultaneously (perceptual condition), or after a 2 s delay (working memory condition). The matching image was the same landscape (and, therefore, contained identical spatial information), but was taken from a different viewpoint and possessed differing non-spatial features. Thus, this task was designed to place a demand on viewpoint independent or allocentric spatial processing (Figure 4). It was found that only two out of four selective hippocampal lesion patients were significantly impaired on the perceptual condition, whereas all four patients struggled in the working memory condition (Hartley et al., 2007). Similarly, there was no significant difference between either a-MCI and $\mathrm{AD}$ patients versus their matched controls on the perceptual task, but significant impairment in both patient groups when working memory demand was increased.

Although the findings from these studies can be used to argue for a role for the hippocampus in spatial working memory but not spatial perception, alternative explanations must also be considered. In the case of Cashdollar et al. (2009), the non-working memory condition involved stimuli that were presented from the same view, and moreover, stimuli that could potentially be discriminated on the basis of a single feature that was absent or spatially moved in the foil stimulus (Cashdollar et al., 2010; Lee and Baxter, 2010). The findings of Hartley et al. (2007) and Bird et al. (2010) are more puzzling, given that (1) the stimuli involved different viewpoints of carefully constructed landscapes, and (2) two out of the four selective hippocampal lesion patients did, in fact, demonstrate significant impairment on the perceptual condition (thus supporting a role for the hippocampus in spatial perception). One possible explanation for the lack of impairment in the remaining selective hippocampal patients and the AD cases is that these patients relied on less flexible non-hippocampal processes to solve the perceptual condition trials successfully (Hartley et al., 2007; Bird et al., 2010), for instance processes that may involve egocentric representations in parahippocampal cortex (Epstein et al., 2003). For example, as the authors acknowledge, it may be that in the perceptual conditions participants with hippocampal damage were able to solve the task by comparing individual features of the scenes using a serial, single feature matching strategy, rather than forming a more holistic representation of the scene. This strategy would be available in the perceptual condition (because all the stimuli and their features were simultaneously presented) but not in the working memory condition (because the target was removed and thus not available for serial single feature comparison).
A

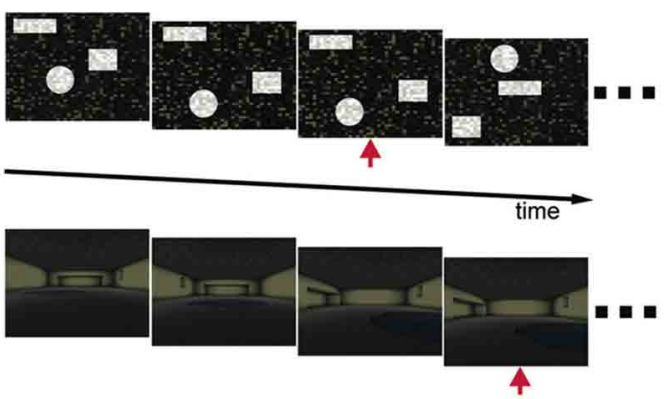

C

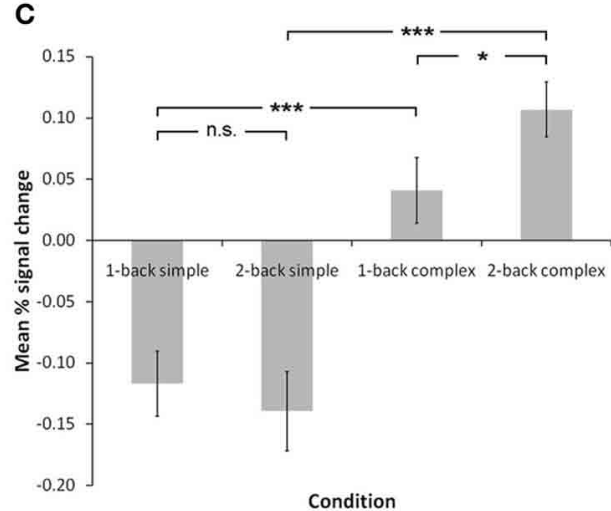

B
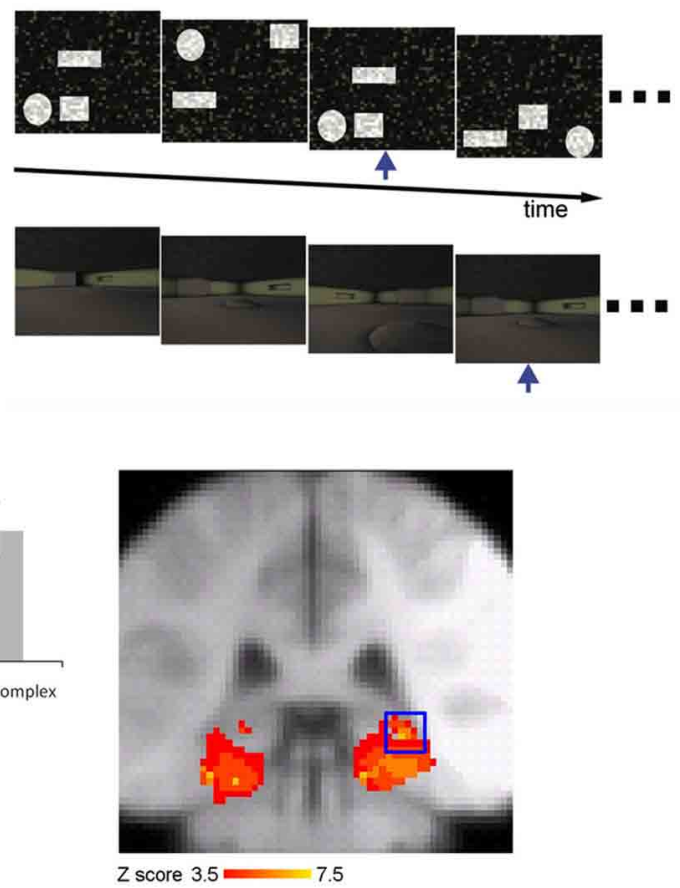

FIGURE 4 | Increasing working memory demand is associated with an increase in hippocampal activity when complex, but not simple, spatial stimuli are presented Lee and Rudebeck (2010b). Participants were required to carry out a one-back $(\mathbf{A})$ or two-back $(\mathbf{B})$ working memory task in an $\mathrm{MRI}$ scanner, involving simple spatial arrays or complex virtual reality rooms (red arrow indicates one-back repetition target, blue arrow indicates two-back repetition target). The pattern of activity observed in the right posterior hippocampus is illustrated in (C) (activity rendered on the MNI152 template at $p<0.05$ family wise error corrected for multiple comparisons with a small volume correction for the medial temporal lobe). 
We have recently examined whether the hippocampus is involved in spatial working memory and/or spatial perception by using fMRI to investigate how differing demands on these processes may influence hippocampal activity (Lee and Rudebeck, 2010b). Participants were scanned while carrying out a 1 - or 2-back working memory task involving simple spatial stimuli (tracking the spatial location of three shapes within a 2-dimensional array) or complex spatial stimuli (tracking the spatial location of three features within a three-dimensional virtual reality room) (Figure 4). Since the simple spatial stimuli were created by scrambling the three-dimensional stimuli, and both sets of stimuli were unfamiliar to the participants, the primary difference between the simple and complex stimuli was that the complex stimuli placed a greater demand on spatial perception by requiring the processing of spatial information within a threedimensional array. In support of the idea that the hippocampus is critical for complex spatial perception, it was found that there was a significant increase in activity in the posterior hippocampus when the complex spatial stimuli were presented in comparison to the simple spatial stimuli, irrespective of 1- vs. 2-back working memory demand (i.e., a main effect of stimulus type). Moreover, there was no region within the MTL that was associated with an increase in activity when working memory demand was increased regardless of stimuli type. Critically, there was an interaction effect in the posterior hippocampus, in which a significant increase in activity was observed when working memory demand was increased, but only when the complex spatial stimuli, and not the simple spatial stimuli, were presented. These data argue that the type of information to be processed is critical to the involvement of the hippocampus in spatial working memory. More specifically, this structure is involved in working memory tasks to the extent that the perception of complex spatial information is necessary (Lee and Rudebeck, 2010b), a suggestion that may account for some of the studies that have not observed working or short-term memory deficits following hippocampal damage (e.g., Murray and Mishkin, 1998; Jeneson et al., 2011).

Further evidence suggesting that spatial discrimination deficits following hippocampal damage can be explained by a spatial perception deficit and not just an impairment in spatial workingmemory comes from the use of eye-tracking to examine saccades during visual discrimination. It is conceivable that any difficulties experienced by patients with MTL lesions in the maintenance and comparison of multiple stimuli in working memory will be reflected in their eye movements between simultaneously presented images. This, however, does not appear to be the case. We have recently examined the eye movements of patients with selective hippocampal lesions and more extensive MTL damage during oddity judgment for different view scenes (as well as other stimulus categories including objects and faces). Crucially, we found that although behavioral performance is compromised, there were no clear differences in the eye movements of the patients compared to controls (Erez et al., in preparation). Of particular interest is the observation that relative to matched controls, patients did not show a reduction in saccades within a given scene as compared to saccades across distinct scenes, a finding that may reflect intact trans-saccadic working memory in the patients.

\section{UNDERSTANDING THE CONTRIBUTION OF THE HIPPOCAMPUS TO COMPLEX SPATIAL PERCEPTION}

One means of gaining insight into how the hippocampus contributes to spatial perception is to consider the types of stimuli that have been shown to elicit perceptual deficits in hippocampal lesion patients. As described previously, deficits in spatial perception have been observed in the spatial oddity task when participants are required to select the odd-one-out from images of scenes presented from different viewpoints (Lee et al., 2005b, 2006). The deficits observed for spatial discrimination tasks involving scenes presented from the same viewpoint are less consistent (Lee et al., 2005b, 2006; Cashdollar et al., 2009; Kim et al., 2011) and furthermore, fMRI work has revealed greater hippocampal activity for different versus same view spatial scene oddity judgment (Barense et al., 2010a). Thus, one obvious conclusion is that the hippocampus contributes to spatial perception via its role in the processing of viewpoint independent or allocentric spatial representations. Given the existence of hippocampal cells that indicate absolute location in rodents, monkeys, and humans (i.e., place cells that fire when an animal is in a specific spatial location irrespective of the direction the animal is facing, O'Keefe, 1976; Ono et al., 1991, 1993; Ekstrom et al., 2003; Hori et al., 2003) and hippocampal cells that signal viewpoint independent representations of locations in monkeys and humans (i.e., spatial view cells, Rolls et al., 1997; Robertson et al., 1998; Ekstrom et al., 2003), the hippocampus has been suggested to be critical for allocentric but not egocentric (i.e., viewpoint dependent) representations (Burgess, 2002) (see Gaffan, 1998, however, for an alternative view). Supporting this idea, hippocampal damage in rodents, monkeys, and humans has been reported to impair performance on spatial memory tasks that place a demand on allocentric spatial representations (Morris et al., 1982, 1990; Holdstock et al., 2000; King et al., 2002, 2004; Lavenex et al., 2006).

Although a role for the hippocampus in processing allocentric spatial representations may account for the spatial discrimination deficits seen during different, but not same, view spatial oddity judgment, it is not immediately obvious how it can explain deficits in the discrimination of spatial scenes that have been blended to create a high level of feature overlap and do not place a demand on allocentric processing (Lee et al., 2005c; see also Lee et al., 2007). The interpretation of these studies was that the hippocampus may contribute to spatial perception by processing distinct complex conjunctions of the features that comprise a spatial scene. Thus, hippocampal damage leads to an impairment in the discrimination of scenes that are perceptually similar or cannot be differentiated easily on the basis of a single spatial feature. To underline this idea, Lee et al. (2005c) found that patients with hippocampal damage made an increasing number of errors in the discrimination of two scene stimuli when the level of blending between the two images was increased.

Converging evidence for the notion that the hippocampus is important for representing conjunctions of spatial information comes from studies examining hippocampal involvement in the discrimination of spatial stimuli of varying complexity (Buckley et al., 2004; Graham et al., 2006; McTighe et al., 2009; Bonnici et al., 2011). For example, Buckley et al. (2004) demonstrated 
that destroying the main output pathway of the hippocampus, the fornix, in monkeys led to deficits in spatial concurrent discrimination learning, but only under some circumstances. Macaque monkeys were trained to discriminate between "tadpole" stimuli (Figure 5), which varied in their position on the screen, angle of tail orientation, and tail length. Although fornix transections did not affect recall of a set of 40 preoperatively acquired spatial concurrent discrimination problems, concurrent learning of new sets of 10 problems was severely impaired. Furthermore, this deficit became markedly worse when the number of foils was increased. Another experiment in the same group of animals found that spatial configural learning was similarly impaired by fornix transections. As shown in Figure $\mathbf{5 A}$, in this task the three elemental features (i.e., position on screen, length, and angle) were maximally overlapping, thus requiring the monkeys to learn a conjunction of these spatial features. Recent studies in rodents have demonstrated that hippocampal dysfunction does not impair all forms of spatial discrimination learning, providing additional evidence that the hippocampus is critical for complex but not simple spatial processing (McHugh et al., 2007, Clelland et al., 2009; McTighe et al., 2009; Talpos et al., 2010). McTighe et al. (2009) used a novel touchscreen paradigm to show that rats with lesions to the dorsal hippocampus were impaired at learning pairs of spatial locations within an array when the spatial

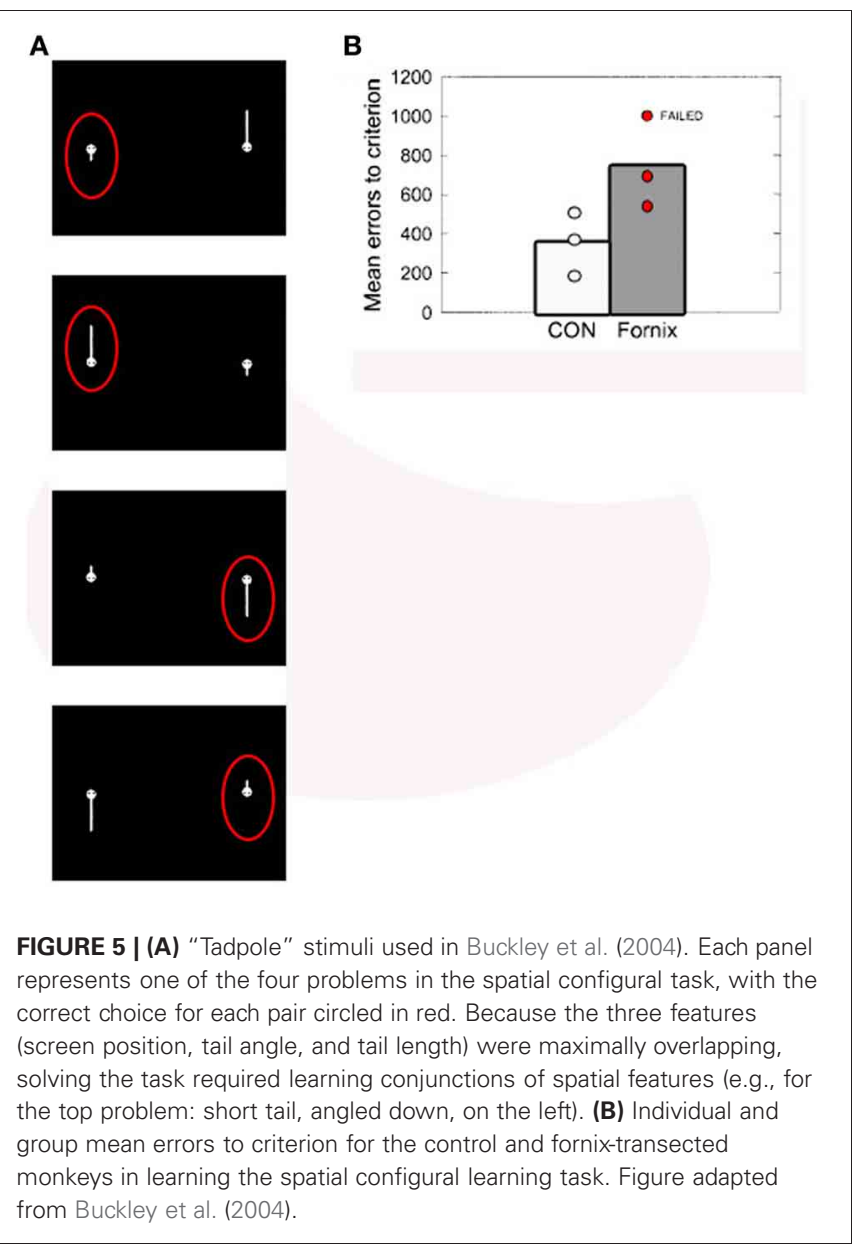

separation between the locations was small (i.e., separated by one location), but not when the separation was large (i.e., separated by 3 or 5 locations). A similar profile of performance was observed by Clelland et al. (2009) in mice in which neurogenesis in the dentate gyrus was ablated through X-ray exposure. Learning demands were consistent across task conditions and only the spatial separation between locations was manipulated. Thus, these findings suggest that the hippocampus is critical in the discrimination of perceptually similar, but not perceptually distinct, spatial stimuli.

Given the perceptual deficits in hippocampal damaged patients in spatial oddity judgment and the discrimination of morphed scene images, and in the light of the respective demands of these two paradigms, it is possible that the hippocampus contributes to spatial perception both via its role in processing allocentric spatial representations, as well as a potential role in processing conjunctions of spatial features. One point to consider is whether these two processes are distinct, overlapping or, in fact, one and the same. For instance, it is conceivable that the ability to perceive and recognize the same scene from multiple viewpoints depends on the ability to successfully process and form a conjunction of the many intricate spatial relationships that exist between the features that make up the scene. Thus, even as the visual appearance of a scene may change across different viewpoints, this conjunction of spatial relationships remains consistent.

If the hippocampus is critical for processing conjunctions of spatial features, one question that arises is how this relates to the role of the PRC and its purported role in processing conjunctions of object features. The representational-hierarchical model (e.g., Saksida and Bussey, 2010) suggests that the hippocampus sits above the PRC at the apex of the representational-hierarchy in the ventral visual stream. Thus, whereas the PRC represents conjunctions of the features that make up an object, the hippocampus represents conjunctions of spatial features or objects (for example those that may comprise a scene), as well as other types of information for which the hippocampus may be critical. One such type of information is likely to be temporal information, with a number of studies highlighting a role for the hippocampus in the processing of sequences and temporal context (Fortin et al., 2002; Kesner et al., 2002; Charles et al., 2004; Jenkins and Ranganath, 2010; Devito and Eichenbaum, 2011; MacDonald et al., 2011; Tubridy and Davachi, 2011).

This idea of higher-level representations in the hippocampus is compatible with the notion that this structure is important for pattern separation and completion (O'Reilly and McClelland, 1994; Treves and Rolls, 1994; Norman and O'Reilly, 2003; Lee et al., 2004; Leutgeb et al., 2005, 2007; Kirwan and Stark, 2007; McHugh et al., 2007; Bakker et al., 2008; Bonnici et al., 2011; Lacy et al., 2011; Yassa and Stark, 2011). Pattern separation refers to the idea that the hippocampus forms distinct representations of the spatial and temporal information that comprise an event, which, therefore, enable similar episodes to be distinguished from one another. On the other hand, pattern completion is suggested to occur when complete episodes can be retrieved on the basis of partial cues. Although we certainly do not disagree with the idea that the hippocampus processes distinct representations of spatial (and other forms of) information that underlie 
the formation and retrieval of memories, we would argue that the purpose and function of these representations is not limited to the mnemonic domain but also extends to perception. Thus, the hippocampus is critical for tasks that place a demand on distinct, complex representations of information (particularly spatial and temporal), regardless of whether these tasks involve different types of long-term memory (e.g., episodic vs. semantic; declarative vs. non-declarative), short-term memory or perceptual processes. In support of this view, a recent fMRI study demonstrated that the recall of spatial information was critical to eliciting hippocampal activity in healthy participants, irrespective of whether the memory task was episodic or semantic in nature (Hoscheidt et al., 2010). Additional support comes from studies that have reported stimulus-specific impairments in patients with hippocampal damage on recognition memory tests (Cipolotti et al., 2006; Taylor et al., 2007; Bird and Burgess, 2008), as well as non-declarative memory tasks that have been traditionally considered to not be dependent on the MTL (Graham et al., 2006 see also Olson et al., this issue). For example, Taylor et al. (2007) found that patients with selective hippocampal damage demonstrated significantly impaired recognition memory for scene, but not, face stimuli, whereas amnesic cases with broader MTL damage incorporating both the hippocampus and MTL were significantly impaired at recognizing both scene and face images. To underline this, Bird et al. (2008) administered a receiver operator characteristic recognition memory task to a developmental amnesic patient with selective hippocampal damage and found impaired scene, but not face, recollection and familiarity memory, suggesting that stimulus type (scenes vs. faces) may be a more critical determinant of hippocampal involvement than mnemonic process (e.g., recollection vs. familiarity; see however, Aly et al., 2010). With regards to non-declarative memory, Graham et al. (2006) tested patients with specific lesions to the hippocampus on a perceptual classification paradigm using faces and scenes. In each trial, participants were shown two exemplars of scenes or faces, and asked to classify a third stimulus (created by morphing together the exemplars with varying levels of feature overlap) as being more similar to one exemplar or the other. Patients with hippocampal lesions demonstrated abnormal performance for both categorization and perceptual learning when the stimuli were virtual reality scenes, but not when the stimuli used were faces. Moreover, their performance declined as the level of feature overlap increased. These findings contradict the idea that the MTL is critical for declarative, but not non-declarative, memory (Squire et al., 2004) and demonstrate that non-declarative memory deficits can be seen following hippocampal damage when spatial stimuli are used (see also, Chun and Phelps, 1999).

In the light of the above, it may be more constructive to consider the functions of the hippocampus with regards to the representations that this structure is critical for, rather than the cognitive processes that it mediates. Thus, although we have been presenting an argument for a role for the hippocampus in spatial perception in this review, we are not suggesting that the hippocampus only subserves spatial perception, or that the hippocampus is not critical for memory. Rather, we argue that the hippocampus represents distinct, complex conjunctions of information (in particular spatial and temporal) that may be recruited for both perceptual and mnemonic processes (for a similar suggestion that the hippocampus is critical for processing unique conjunctions of stimulus elements see Aggleton et al., 2007). From this perspective, one may begin to see how a representational account of hippocampal function relates to other mnemonic theories of MTL function and the evidence on which these theories are based (i.e., recollection vs. familiarity; relational vs. non-relational memory) (see also Bird and Burgess, 2008). For instance, recollection memory involves the recall of rich contextual information, of which spatial and/or temporal information is typically a critical component. Hippocampal damage, therefore, may disrupt the complex representations that are necessary for this contextual recall, leading to the observation of a significant deficit in recollection, but not familiarity, memory (e.g., Yonelinas et al., 2002; Fortin et al., 2004; Aggleton et al., 2005; Vann et al., 2009). As described earlier, however, intact recollection can be seen after hippocampal damage if the stimuli to be remembered do not place a significant demand on these hippocampal-dependent representations (Bird et al., 2008). With regards to the theory that relational memory is dependent on the hippocampus, we would argue that (1) the complex hippocampal representations that we have described can underlie relational memory processing but importantly, also contribute to relational processing in the context of tasks that do not have a mnemonic demand; and (2) the nature of these hippocampal representations suggests that the hippocampus does not subserve all forms of relational processing. Studies of relational memory in humans have often involved testing memory for the relation of an object or word and its given location or context (e.g., Davachi et al., 2003; Giovanello et al., 2004; Ryan and Cohen, 2004; Hannula et al., 2006; Olson et al., 2006b; Hannula and Ranganath, 2008). These tests have, therefore, inherently assessed relational memory for stimuli embedded with spatial information. There is evidence to suggest that the hippocampus is not necessary for all forms of relational processing, or at least is recruited to a greater extent for spatial vs. non-spatial relationships. Functional neuroimaging work has demonstrated significant hippocampal involvement when spatial relationships are considered, but not when social relationships between individuals are processed (Kumaran and Maguire, 2005). Along similar lines, although hippocampal activity has been observed for both spatial and non-spatial relational memory, the former has been associated with significantly greater hippocampal activity (Ryan et al., 2010). In addition to this, a recent study reported intact associative memory for different items within the same stimulus category (e.g., faces or words) in a patient with selective hippocampal damage (Mayes et al., 2004). This same patient, on the other hand, showed clear recognition memory deficits for associations between different types of information (e.g., words and locations), with many of the associative memory tests either assessing memory for objects and spatial location or their temporal position (see, however, Mayes et al., 2007 for discussion on unitization of items in memory).

Our suggestion that information type is a critical determinant of hippocampal involvement in any given cognitive task is shared by other theories of MTL function. In particular, the BIC model (Diana et al., 2007; Eichenbaum et al., 2007; Ranganath, 2010) argues that the hippocampus is important for the binding 
together of item and context information: this type of information is critical for recollective memory but importantly, may also be necessary for short-term memory tasks, perception or nonconscious memory depending on the type of stimuli that are involved. Where our ideas may diverge is that whereas we are suggesting that the hippocampus is critical for representing conjunctions of spatial and temporal information, the BIC model defines the notion of context more broadly, potentially incorporating conceptual or semantic frameworks, background features in a visual image or even objects themselves (Ranganath, 2010).

Broadly speaking, although cognitive mnemonic theories of MTL function have provided and continue to provide valuable insight into MTL-dependent processing, they cannot account easily for a growing body of evidence implicating a role for the MTL in complex visual discrimination. By shaping our understanding of the different MTL structures (i.e., hippocampus and PRC) with regards to representations (Murray et al., 2007; Graham et al., 2010; Saksida and Bussey, 2010) rather than cognitive processes, it may be possible to understand how these structures can contribute to both memory and higher-order perception and thus, reconcile data from visual discrimination tasks to findings from paradigms assessing mnemonic processing.

\section{CONCLUSIONS AND FUTURE DIRECTIONS}

In summary, recent work has demonstrated that amnesic patients with hippocampal damage exhibit significant impairments in the discrimination of simultaneously presented complex spatial scene stimuli. We argue that these deficits are unlikely to be a result of a primary impairment in incremental learning, incidental encoding into long-term memory or short-term working memory. Moreover, these visual discrimination difficulties cannot be explained easily by unreported structural damage or dysfunction beyond the MTL. Instead, we suggest that these findings reflect a role for the hippocampus in higher-order spatial perception, with this structure being critical for the representation of complex conjunctions of features that constitute spatial scenes. As has been suggested elsewhere (e.g., Saksida and Bussey, 2010; Cowell et al., 2010a), these representations are likely to encompass or interact with other types of information for which the hippocampus may be important, such as temporal information. Thus, these representations may not only underlie a role for the hippocampus in episodic memory (e.g., via mechanisms such as pattern separation and completion), but also other areas of cognition (e.g., perception, non-declarative memory) provided that the task at hand places a sufficient demand on these representations, for example by means of the types of stimuli that they involve. We argue that the role of the hippocampus should be considered not in terms of broad psychological constructs, but in terms of neural representations and computational mechanisms. These representations and mechanisms are shared across several cognitive processes. Damage to the hippocampus, therefore, impairs performance on a variety of seemingly disparate cognitive tasks.

One necessary aim for future research is to understand the nature and precise composition of these hippocampal representations. More specifically, although we have suggested that the hippocampus represents complex conjunctions of spatial features, it is unclear what exactly these features are and what neural and computational mechanisms create these representations. One obvious candidate that may be central to clarifying these issues is the hippocampal place cell, given its well established role in spatial cognition (O'Keefe et al., 1998; Best et al., 2001; Moser et al., 2008). Indeed, the suggestion that the hippocampus is critical for distinct spatial representations is consistent (and perhaps in some respects even synonymous) with the notion that the hippocampus functions as a cognitive map (O'Keefe and Nadel, 1978). The electrophysiological properties of place cells have been suggested to reflect or be a product of afferent information (Burgess et al., 2000; Hartley et al., 2000; O’Keefe and Burgess, 2005; Barry et al., 2006; McNaughton et al., 2006; Rolls et al., 2006), including that from boundary vector cells in the subiculum (Lever et al., 2009), which are believed to signal the distance and directional vector to an environmental boundary, and entorhinal grid cells (Fyhn et al., 2004), which are similar to place cells except that each cell has multiple firing fields organized in a grid-like formation. Moreover, place cells may be able to provide a code for temporal information by means of theta phase precession (O'Keefe and Recce, 1993; Huxter et al., 2003), the phenomenon in which a place cell fires at increasingly earlier phases of the theta rhythm when an animal traverses the associated place field. More recently, hippocampal cells that encode successive moments in an empty delay period between two successive events have been discovered in rats (i.e., encountering two objects, followed by encountering two odors) (MacDonald et al., 2011). These "time cells" may work in concert with place cells to provide the spatial and temporal information that constitute the complex representations for which we are suggesting the hippocampus is critical. One area of uncertainty, however, is whether the type of spatial information that is conveyed by place cells (i.e., spatial location) is necessary for some of the spatial discrimination tasks that have been shown to be hippocampal-dependent. For instance, as discussed in the previous section, it is not obvious how allocentric representations of spatial location are related to the ability to discriminate two highly similar spatial scene images that have been created by blending two exemplar scenes to different degrees (Lee et al., 2005c, 2007). This raises the question, therefore, of what other types of spatial features necessary for the perception of scenes are processed by the hippocampus, and what the corresponding underlying neural mechanisms are.

A related question that future research needs to address is how these spatial features are bound together with temporal information to create the complex hippocampal representations that we describe. Within the mnemonic domain, the hippocampus has been shown to be important for binding events or information across time and/or space (e.g., Rawlins, 1985; Qin et al., 2007; Staresina and Davachi, 2009), and it has been suggested that specific neural circuitry and mechanisms within the hippocampus (e.g., recurrent neuronal network organization in CA3; hippocampal theta oscillations) may underlie this function (e.g., Wallenstein et al., 1998; Lisman, 1999). It is unclear whether the same circuitry/mechanisms mediate the formation of complex hippocampal-dependent spatiotemporal representations, or whether there are alternative mechanisms that operate at a different spatial and/or temporal scale to create 
these representations that can subserve both perceptual and mnemonic processes.

A third goal for future work is to understand how a disruption to complex conjunctions of spatial (and temporal) information in the hippocampus (e.g., as a result of structural brain damage) may contribute to or cause deficits in memory. On the basis of computational modeling and empirical findings, the representationalhierarchical model makes clear predictions about the impact of PRC damage on object recognition memory for trial-unique objects (Cowell et al., 2006; Bartko et al., 2010; McTighe et al., 2010). In a standard test of object recognition memory, delayedmatching-to-sample, a sample object is presented, followed by a target and a unique foil object after an intervening delay period. During this delay period, an individual is often exposed to objects (real or imagined) that share many lower-level features with the target-merely by virtue of the fact that the objects in our environment have many features in common (e.g., shapes, colors, etc.). These repeating features lead to massive interference at the level of the individual features, and at the feature-level, all the features appear to be familiar. However, because the objects themselves are trial-unique, the higher-level complex conjunctive representations in PRC can resolve this feature-level interference. In the absence of these conjunctive representations, the judgment of prior occurrence must be on the basis of individual object features, and thus, PRC damage impairs performance (McTighe et al., 2010; Saksida and Bussey, 2010). A similar mechanism may be operating in the hippocampus. Cowell et al. (2006) suggest that when objects repeat in a delayed-matching-to-sample task (i.e., the objects are not trial-unique), the solution requires an even more complex representation than that contained in the PRC because in order to disambiguate the repeating objects, each must be associated with spatial and/or temporal information (Cowell et al., 2010a). Beyond the delayed-matching-to-sample paradigm, it is possible that these complex representations of spatial and

\section{REFERENCES}

Aggleton, J. P., and Brown, M. W. (1999). Episodic memory, amnesia and the hippocampal-anterior thalamic axis. Behav. Brain Sci. 22, 425-289.

Aggleton, J. P., Sanderson, D. J., and Pearce, J. M. (2007). Structural learning and the hippocampus. Hippocampus 17, 723-734.

Aggleton, J. P., Vann, S. D., Denby, C., Dix, S., Mayes, A. R., Roberts, N., and Yonelinas, A. P. (2005). Sparing of the familiarity component of recognition memory in a patient with hippocampal pathology. Neuropsychologia 43, 1810-1823.

Agster, K. L., Fortin, N. J., and Eichenbaum, H. (2002). The hippocampus and disambiguation of overlapping sequences. J. Neurosci. 22, 5760-5768.

Aly, M., Knight, R. T., and Yonelinas, A. P. (2010). Faces are special but not too special: spared face recognition

temporal information enable the disambiguation of repeating or overlapping events. As we have argued here, conjunctions of this type are thought to be processed in the hippocampus. Consistent with this hypothesis, lesions of the hippocampus or fornix in rats are known to impair performance on a delayed-matching-tosample task with repeating items (Rawlins et al., 1993). Moreover, the hippocampus is known to be involved in the processing of overlapping sequences and events (e.g., Levy, 1996; Wood et al., 2000; Agster et al., 2002; Kumaran and Maguire, 2006a). Even if this suggestion is supported by future studies, it still remains to be established how these complex hippocampal representations can extend to mnemonic processing in the context of other memory tasks, and whether the additional consideration of temporal information is sufficient to account for all of the memory deficits that are observed following hippocampal damage. To date, a number of well-conceived theories and computational models have linked spatial cognition and memory, with a number of these placing an emphasis on the importance of allocentric representations of location (e.g., Barry et al., 2006; Bird and Burgess, 2008). As future experimental and computational work reveals further details about the spatial processes that are necessary to perform the spatial discrimination tasks shown to require the hippocampus (Lee et al., 2005b,c, 2006, 2007; Hartley et al., 2007), we should have a clearer grasp of how our suggestion that the hippocampus represents complex conjunctions of spatial features relates to these other models, and how these representations may underlie mnemonic processing.

\section{ACKNOWLEDGMENTS}

The authors are supported by grants from the Natural Sciences and Engineering Research Council of Canada (Discovery Grants to Andy C. H. Lee, Morgan D. Barense; Discovery Accelerator Supplement to Andy C. H. Lee) and the Canadian Institutes of Health Research (Morgan D. Barense).

of viewpoint. Hippocampus 20, 389-401.

Barense, M. D., Rogers, T. T., Bussey, T. J., Saksida, L. M., and Graham, K. S. (2010b). Influence of conceptual knowledge on visual object discrimination: insights from semantic dementia and mtl amnesia. Cereb. Cortex 1-15.

Barense, M. D., Henson, R. N., and Graham, K. S. (2011a). Perception and conception: temporal lobe activity during complex discriminations of familiar and novel faces and objects. J. Cogn. Neurosci. 23, 3052-3067.

Barense, M. D., Ngo, J. K., Hung, L. H., and Peterson, M. A. (2011b). Interactions of memory and perception in amnesia: the figureground perspective. Cereb. Cortex. doi: 10.1093/cercor/bhr347. [Epub ahead of print].

Barry, C., Lever, C., Hayman, R., Hartley, T., Burton, S., O'Keefe, J.,
Jeffery, K., and Burgess, N. (2006). The boundary vector cell model of place cell firing and spatial memory. Rev. Neurosci. 17, 71-97.

Bartko, S. J., Cowell, R. A., Winters, B. D., Bussey, T. J., and Saksida, L. M. (2010). Heightened susceptibility to interference in an animal model of amnesia: impairment in encoding, storage, retrieval-or all three? Neuropsychologia 48, 2987-2997.

Bartko, S. J., Winters, B. D., Cowell, R. A., Saksida, L. M., and Bussey, T. J. (2007a). Perceptual functions of perirhinal cortex in rats: zerodelay object recognition and simultaneous oddity discriminations. $J$. Neurosci. 27, 2548-2559.

Bartko, S. J., Winters, B. D., Cowell, R. A., Saksida, L. M., and Bussey, T. J. (2007b). Perirhinal cortex resolves feature ambiguity in configural object recognition and perceptual oddity tasks. Learn. Mem. 14, 821-832. 
Baxter, M. G. (2009). Involvement of medial temporal structures in memory and perception. Neuron 61, 667-677.

Best, P. J., White, A. M., and Minai, A. (2001). Spatial processing in the brain: the activity of hippocampal place cells. Annu. Rev. Neurosci. 24, 459-486.

Bird, C. M., and Burgess, N. (2008). The hippocampus and memory: insights from spatial processing. Nat. Rev. Neurosci. 9, 182-194.

Bird, C. M., Chan, D., Hartley, T., Pijnenburg, Y. A., Rossor, M. N., and Burgess, N. (2010). Topographical short-term memory differentiates Alzheimer's disease from frontotemporal lobar degeneration. Hippocampus 20, 1154-1169.

Bird, C. M., Vargha-Khadem, F., and Burgess, N. (2008). Impaired memory for scenes but not faces in developmental hippocampal amnesia: a case study. Neuropsychologia 46, 1050-1059.

Bohbot, V. D., Kalina, M., Stepankova, K., Spackova, N., Petrides, M., and Nadel, L. (1998). Spatial memory deficits in patients with lesions to the right hippocampus and to the right parahippocampal cortex. Neuropsychologia 36, 1217-1238.

Bonnici, H. M., Kumaran, D., Chadwick, M. J., Weiskopf, N., Hassabis, D., and Maguire, E. A. (2011). Decoding representations of scenes in the medial temporal lobes. Hippocampus. doi: 10.1002/hipo.20960. [Epub ahead of print].

Brown, M. W., and Aggleton, J. P. (2001). Recognition memory: what are the roles of the perirhinal cortex and hippocampus? Nat. Rev. Neurosci. 2, 51-61.

Buckley, M. J., Booth, M. C., Rolls, E. T., and Gaffan, D. (2001). Selective perceptual impairments after perirhinal cortex ablation. J. Neurosci. 21 , 9824-9836.

Buckley, M. J., Charles, D. P., Browning, P. G., and Gaffan, D. (2004). Learning and retrieval of concurrently presented spatial discrimination tasks: role of the fornix. Behav. Neurosci. 118, 138-149.

Buckley, M. J., and Gaffan, D. (1997). Impairment of visual objectdiscrimination learning after perirhinal cortex ablation. Behav. Neurosci. 111, 467-475.

Buckley, M. J., and Gaffan, D. (1998). Perirhinal cortex ablation impairs visual object identification. $J$. Neurosci. 18, 2268-2275.

Buckley, M. J., and Gaffan, D. (2006). Perirhinal cortical contributions to object perception. Trends Cogn. Sci. $10,100-107$.

Burgess, N. (2002). The hippocampus, space, and viewpoints in episodic memory. Q. J. Exp. Psychol. A 55, 1057-1080.

Burgess, N., Jackson, A., Hartley, T., and O'Keefe, J. (2000). Predictions derived from modelling the hippocampal role in navigation. Biol. Cybern. 83, 301-312.

Burgess, N., Maguire, E. A., and O'Keefe, J. (2002). The human hippocampus and spatial and episodic memory. Neuron 35, 625-641.

Bussey, T. J., and Saksida, L. M. (2002). The organization of visual object representations: a connectionist model of effects of lesions in perirhinal cortex. Eur. J. Neurosci. 15, 355-364.

Bussey, T. J., and Saksida, L. M. (2007). Memory, perception, and the ventral visual-perirhinal-hippocampal stream: thinking outside of the boxes. Hippocampus 17, 898-908.

Bussey, T. J., Saksida, L. M., and Murray, E. A. (2002). Perirhinal cortex resolves feature ambiguity in complex visual discriminations. Eur. J. Neurosci. 15, 365-374.

Bussey, T. J., Saksida, L. M., and Murray, E. A. (2003). Impairments in visual discrimination after perirhinal cortex lesions: testing "declarative" vs. "perceptual-mnemonic" views of perirhinal cortex function. Eur. J. Neurosci. 17, 649-660.

Bussey, T. J., Saksida, L. M., and Murray, E. A. (2005). The perceptual-mnemonic/feature conjunction model of perirhinal cortex function. Q. J. Exp. Psychol. B 58, 269-282.

Cashdollar, N., Lavie, N., and Duzel, E. (2010). Reply to Lee and Baxter: perceptual deficits cannot explain impaired configural-relational maintenance in bilateral hippocampal injury. Proc. Natl. Acad. Sci. U.S.A. 107, E22-E22.

Cashdollar, N., Malecki, U., RuggGunn, F. J., Duncan, J. S., Lavie, N., and Duzel, E. (2009). Hippocampus-dependent and -independent theta-networks of active maintenance. Proc. Natl. Acad. Sci. U.S.A. 106, 20493-20498.

Charles, D. P., Gaffan, D., and Buckley, M. J. (2004). Impaired recency judgments and intact novelty judgments after fornix transection in monkeys. J. Neurosci. 24, 2037-2044.

Chun, M. M., and Phelps, E. A. (1999). Memory deficits for implicit contextual information in amnesic subjects with hippocampal damage. Nat. Neurosci. 2, 844-847.
Cipolotti, L., Bird, C., Good, T., Macmanus, D., Rudge, P., and Shallice, T. (2006). Recollection and familiarity in dense hippocampal amnesia: a case study Neuropsychologia 44, 489-506.

Clark, R. E., Reinagel, P., Broadbent, N. J., Flister, E. D., and Squire, L. R. (2011). Intact performance on feature-ambiguous discriminations in rats with lesions of the perirhinal cortex. Neuron 70, 132-140

Clelland, C. D., Choi, M., Romberg, C., Clemenson, G. D. Jr., Fragniere, A. Tyers, P., Jessberger, S., Saksida, L. M., Barker, R. A., Gage, F. H., and Bussey, T. J. (2009). A functional role for adult hippocampal neurogenesis in spatial pattern separation. Science 325, 210-213.

Cohen, N. J., and Eichenbaum, H. (1993). Memory, Amnesia, and The Hippocampal System Massachusetts, MA: MIT Press.

Cohen, N. J., and Squire, L. R. (1980). Preserved learning and retention of pattern-analyzing skill in amnesia: dissociation of knowing how and knowing that. Science 210, 207-210.

Cowell, R. A., Bussey, T. J., and Saksida L. M. (2006). Why does brain damage impair memory? A connectionist model of object recognition memory in perirhinal cortex. J. Neurosci. 26, 12186-12197.

Cowell, R. A., Bussey, T. J., and Saksida, L. M. (2010a). Components of recognition memory: dissociable cognitive processes or just differences in representational complexity? Hippocampus 20 1245-1262.

Cowell, R. A., Bussey, T. J., and Saksida, L. M. (2010b). Functional dissociations within the ventral object processing pathway: cognitive modules or a hierarchical continuum? J. Cogn. Neurosci. 22, 2460-2479.

Davachi, L., Mitchell, J. P., and Wagner, A. D. (2003). Multiple routes to memory: distinct medial temporal lobe processes build item and source memories, Proc Natl. Acad. Sci. U.S.A. 100, 2157-2162.

Davies, R. R., Graham, K. S., Xuereb, J. H., Williams, G. B., and Hodges, J. R. (2004). The human perirhina cortex and semantic memory. Eur. J. Neurosci. 20, 2441-2446.

Devito, L. M., and Eichenbaum, H. (2011). Memory for the order of events in specific sequences: contributions of the hippocampus and medial prefrontal cortex. J. Neurosci. 31, 3169-3175.

Devlin, J. T., and Price, C. J. (2007). Perirhinal contributions to human visual perception. Curr. Biol. 17, 1484-1488
Diana, R. A., Yonelinas, A. P., and Ranganath, C. (2007). Imaging recollection and familiarity in the medial temporal lobe: a threecomponent model. Trends Cogn. Sci. 11, 379-386.

Eacott, M. J., Gaffan, D., and Murray, E. A. (1994). Preserved recognition memory for small sets, and impaired stimulus identification for large sets, following rhinal cortex ablations in monkeys. Eur. J. Neurosci. 6, 1466-1478.

Eichenbaum, H., Otto, T., and Cohen, N. J. (1992). The hippocampuswhat does it do? Behav. Neural Biol. $57,2-36$.

Eichenbaum, H., Yonelinas, A. P., and Ranganath, C. (2007). The medial temporal lobe and recognition memory. Annu. Rev. Neurosci. 30, 123-152.

Ekstrom, A. D., Kahana, M. J., Caplan, J. B., Fields, T. A., Isham, E. A., Newman, E. L., and Fried, I. (2003). Cellular networks underlying human spatial navigation. Nature 425, 184-188.

Epstein, R., Graham, K. S., and Downing, P. E. (2003). Viewpointspecific scene representations in human parahippocampal cortex Neuron 37, 865-876.

Epstein, R. A., and Kanwisher, N. (1998). A cortical representation of the local visual environment. Nature 392, 598-601.

Feigenbaum, J. D., and Morris, R. G. (2004). Allocentric versus egocentric spatial memory after unilateral temporal lobectomy in humans. Neuropsychology 18, 462-472.

Fortin, N. J., Agster, K. L., and Eichenbaum, H. B. (2002). Critical role of the hippocampus in memory for sequences of events. Nat. Neurosci. 5, 458-462.

Fortin, N. J., Wright, S. P., and Eichenbaum, H. (2004). Recollection-like memory retrieval in rats is dependent on the hippocampus. Nature 431, 188-191.

Fyhn, M., Molden, S., Witter, M. P., Moser, E. I., and Moser, M. B. (2004). Spatial representation in the entorhinal cortex. Science 305, 1258-1264.

Gaffan, D. (1998). Idiothetic input into object-place configuration as the contribution to memory of the monkey and human hippocampus: a review. Exp. Brain Res. 123, 201-209.

Gaffan, D. G. (2001). What is a memory system? Horel's critique revisited. Behav. Brain Res. 127, 5-11.

Gaffan, D. G. (2002). Against memory systems. Philos. Trans. R Soc. B Biol. Sci. 357, 1111-1121. 
Galton, C. J., Gomez-Anson, B., Antoun, N., Scheltens, P., Patterson, K., Graves, M., Sahakian, B. J., and Hodges, J. R. (2001). Temporal lobe rating scale: application to Alzheimer's disease and frontotemporal dementia. J. Neurol. Neurosurg. Psychiatry 70, 165-173.

Giovanello, K. S., Schnyer, D. M., and Verfaellie, M. (2004). A critical role for the anterior hippocampus in relational memory: evidence from an fMRI study comparing associative and item recognition. Hippocampus 14, 5-8.

Graham, K. S., Barense, M. D., and Lee, A. C. (2010). Going beyond LTM in the MTL: a synthesis of neuropsychological and neuroimaging findings on the role of the medial temporal lobe in memory and perception. Neuropsychologia 48, 831-853.

Graham, K. S., Scahill, V. L., Hornberger, M., Barense, M. D., Lee, A. C., Bussey, T. J., and Saksida, L. M. (2006). Abnormal categorization and perceptual learning in patients with hippocampal damage. J. Neurosci. 26, 7547-7554.

Hampton, R. R., Hampstead, B. M., and Murray, E. A. (2004). Selective hippocampal damage in rhesus monkeys impairs spatial memory in an open-field test. Hippocampus $14,808$.

Hannula, D. E., and Ranganath, C. (2008). Medial temporal lobe activity predicts successful relational memory binding. J. Neurosci. 28, 116-124.

Hannula, D. E., Tranel, D., and Cohen, N. J. (2006). The long and the short of it: relational memory impairments in amnesia, even at short lags. J. Neurosci. 26, 8352-8359.

Hartley, T., Bird, C. M., Chan, D., Cipolotti, L., Husain, M., VarghaKhadem, F., and Burgess, N. (2007). The hippocampus is required for short-term topographical memory in humans. Hippocampus 17, 34-48.

Hartley, T., Burgess, N., Lever, C., Cacucci, F., and O'Keefe, J. (2000). Modeling place fields in terms of the cortical inputs to the hippocampus. Hippocampus 10, 369-379.

Hassabis, D., Chu, C., Rees, G., Weiskopf, N., Molyneux, P. D., and Maguire, E. A. (2009). Decoding neuronal ensembles in the human hippocampus. Curr. Biol. 19, 1-9.

Holdstock, J. S., Mayes, A. R., Cezayirli, E., Isaac, C. L., Aggleton, J. P., and Roberts, N. (2000). A comparison of egocentric and allocentric spatial memory in a patient with selective hippocampal damage. Neuropsychologia 38, 410-425.
Hori, E., Tabuchi, E., Matsumura, N., Tamura, R., Eifuku, S., Endo, S., Nishijo, H., and Ono, T. (2003). Representation of place by monkey hippocampal neurons in real and virtual translocation. Hippocampus 13, 190-196.

Hoscheidt, S. M., Nadel, L., Payne, J., and Ryan, L. (2010). Hippocampal activation during retrieval of spatial context from episodic and semantic memory. Behav. Brain Res. 212, 121-132.

Huxter, J., Burgess, N., and O'Keefe, J. (2003). Independent rate and temporal coding in hippocampal pyramidal cells. Nature 425, 828-832.

Igloi, K., Doeller, C. F., Berthoz, A., Rondi-Reig, L., and Burgess, N. (2010). Lateralized human hippocampal activity predicts navigation based on sequence or place memory. Proc. Natl. Acad. Sci. U.S.A. 107, 14466-14471.

Jeneson, A., Mauldin, K. N., Hopkins, R. O., and Squire, L. R. (2011). The role of the hippocampus in retaining relational information across short delays: the importance of memory load. Learn. Mem. 18, 301-305.

Jenkins, L. J., and Ranganath, C. (2010). Prefrontal and medial temporal lobe activity at encoding predicts temporal context memory. J. Neurosci. 30, 15558-15565.

Kanwisher, N., McDermott, J., and Chun, M. M. (1997). The fusiform face area: a module in human extrastriate cortex specialized for face perception. J. Neurosci. 17, 4302-4311.

Kesner, R. P., Gilbert, P. E., and Barua, L. A. (2002). The role of the hippocampus in memory for the temporal order of a sequence of odors. Behav. Neurosci. 116, 286-290.

Kim, S., Jeneson, A., Van Der Horst, A. S., Frascino, J. C., Hopkins, R. O., and Squire, L. R. (2011). Memory, visual discrimination performance, and the human hippocampus. J. Neurosci. 31, 2624-2629.

King, J. A., Burgess, N., Hartley, T., Vargha-Khadem, F., and O'Keefe, J. (2002). Human hippocampus and viewpoint dependence in spatial memory. Hippocampus 12, 811-820.

King, J. A., Trinkler, I., Hartley, T., Vargha-Khadem, F., and Burgess, N. (2004). The hippocampal role in spatial memory and the familiarityrecollection distinction: a case study. Neuropsychology 18, 405-417.

Kirwan, C. B., and Stark, C. E. (2007). Overcoming interference: an fMRI investigation of pattern separation in the medial temporal lobe. Learn. Mem. 14, 625-633.

Kourtzi, Z., and Kanwisher, N. (2000). Cortical regions involved in perceiving object shape. J. Neurosci. 20, 3310-3318.

Kumaran, D., and Maguire, E. A. (2005). The human hippocampus: cognitive maps or relational memory? J. Neurosci. 25, 7254-7259.

Kumaran, D., and Maguire, E. A (2006a). The dynamics of hippocampal activation during encoding of overlapping sequences. Neuron 49, 617-629.

Kumaran, D., and Maguire, E. A. (2006b). An unexpected sequence of events: mismatch detection in the human hippocampus. PLoS Biol. 4:e424. doi: 10.1371/journal.pbio.0040424

Kumaran, D., and Maguire, E. A. (2007). Match mismatch processes underlie human hippocampal responses to associative novelty. $J$. Neurosci. 27, 8517-8524.

Lacy, J. W., Yassa, M. A., Stark, S. M., Muftuler, L. T., and Stark, C. E. (2011). Distinct pattern separation related transfer functions in human CA3/dentate and CAl revealed using high-resolution $\mathrm{AMRI}$ and variable mnemonic similarity. Learn. Mem. 18, 15-18.

Lavenex, P. B., Amaral, D. G., and Lavenex, P. (2006). Hippocampal lesion prevents spatial relational learning in adult macaque monkeys. J. Neurosci. 26, 4546-4558.

Lee, A. C. H., Barense, M. D., and Graham, K. S. (2005a). The contribution of the human medial temporal lobe to perception: bridging the gap between animal and human studies. Q. J. Exp. Psychol. B 58, 300-325.

Lee, A. C. H., and Baxter, M. G. (2010). Hippocampus and configural-relational information: a relationship confined to memory? Proc. Natl. Acad. Sci. U.S.A. 107, E21-E22.

Lee, A. C. H., Buckley, M. J., Gaffan, D., Emery, T., Hodges, J. R., and Graham, K. S. (2006). Differentiating the roles of the hippocampus and perirhinal cortex in processes beyond long-term declarative memory: a double dissociation in dementia. J. Neurosci. 26, 5198-5203.

Lee, A. C. H., Buckley, M. J., Pegman, S. J., Spiers, H., Scahill, V. L., Gaffan, D., Bussey, T. J., Davies, R. R., Kapur N., Hodges, J. R., and Graham, K. S. (2005b). Specialisation in the medial temporal lobe for processing of objects and scenes. Hippocampus $15,782-797$.
Lee, A. C. H., Bussey, T. J., Murray, E. A., Saksida, L. M., Epstein, R. A., Kapur, N., Hodges, J. R., and Graham, K. S. (2005c). Perceptual deficits in amnesia: challenging the medial temporal lobe "mnemonic" view. Neuropsychologia 43, 1-11.

Lee, A. C. H., Gough, K., Davies, R. R., Barense, M. D., and Graham, K. S. (2008). The medial temporal lobe and processes beyond long-term memory: a volumetric MRI study. Soc. Neurosci. Abstr. 785, 1.

Lee, A. C. H., Levi, N., Davies, R. R., Hodges, J. R., and Graham, K. S. (2007). Differing profiles of face and scene discrimination deficits in semantic dementia and Alzheimer's disease. Neuropsychologia 45, 2135-2146.

Lee, A. C. H., and Rudebeck, S. R. (2010a). Human medial temporal lobe damage can disrupt the perception of single objects. J. Neurosci. 30, 6588-6594.

Lee, A. C. H., and Rudebeck, S. R. (2010b). Investigating the interaction between spatial perception and working memory in the human medial temporal lobe. J. $\operatorname{Cog} n$. Neurosci. 22, 2823-2835.

Lee, A. C. H., Scahill, V. L., and Graham, K. S. (2008). Activating the medial temporal lobe during oddity judgment for faces and scenes. Cereb. Cortex 18, 683-696.

Lee, I., Yoganarasimha, D., Rao, G., and Knierim, J. J. (2004). Comparison of population coherence of place cells in hippocampal subfields CA1 and CA3. Nature 430, 456-459.

Leutgeb, J. K., Leutgeb, S., Moser, M. B., and Moser, E. I. (2007). Pattern separation in the dentate gyrus and CA3 of the hippocampus. Science 315, 961-966.

Leutgeb, S., Leutgeb, J. K., Barnes, C. A., Moser, E. I., McNaughton, B. L., and Moser, M.-B. (2005). Independent codes for spatial and episodic memory in hippocampal neuronal ensembles. Science 309, 619-623.

Lever, C., Burton, S., Jeewajee, A., O'Keefe, J., and Burgess, N. (2009). Boundary vector cells in the subiculum of the hippocampal formation. J. Neurosci. 29, 9771-9777.

Levy, D. A., Shrager, Y., and Squire, L. R. (2005). Intact visual discrimination of complex and feature-ambiguous stimuli in the absence of perirhinal cortex. Learn. Mem. 12, 61-66.

Levy, W. B. (1996). A sequence predicting CA3 is a flexible associator that learns and uses context to solve hippocampal-like tasks. Hippocampus 6, 579-590. 
Lisman, J. E. (1999). Relating hippocampal circuitry to function: recall of memory sequences by reciprocal dentate-CA3 interactions. Neuron 22, 233-242.

MacDonald, C. J., Lepage, K. Q., Eden, U. T., and Eichenbaum, H. (2011). Hippocampal "time cells" bridge the gap in memory for discontiguous events. Neuron 71, 737-749.

Maguire, E. A., Burgess, N., Donnett, J. G., Frackowiak, R. S., Frith, C. D., and O'Keefe, J. (1998). Knowing where and getting there: a human navigation network. Science 280, 921-924.

Maguire, E. A., Frackowiak, R. S., and Frith, C. D. (1997). Recalling routes around london: activation of the right hippocampus in taxi drivers. J. Neurosci. 17, 7103-7110.

Maguire, E. A., Nannery, R., and Spiers, H. J. (2006). Navigation around London by a taxi driver with bilateral hippocampal lesions. Brain 129, 2894-2907.

Malach, R., Reppas, J. B., Benson, R. R., Kwong, K. K., Jiang, H., Kennedy, W. A., Ledden, P. J., Brady, T. J., Rosen, B. R., and Tootell, R. B. (1995). Object-related activity revealed by functional magnetic resonance imaging in human occipital cortex. Proc. Natl. Acad. Sci. U.S.A. 92, 8135-8139.

Mayes, A. R., Holdstock, J. S., Isaac, C. L., Montaldi, D., Grigor, J., Gummer, A., Cariga, P., Downes, J. J., Tsivilis, D., Gaffan, D., Gong, Q., and Norman, K. A. (2004). Associative recognition in a patient with selective hippocampal lesions and relatively normal item recognition. Hippocampus 14, 763-784.

Mayes, A. R., Montaldi, D., and Migo, E. (2007). Associative memory and the medial temporal lobes. Trends Cogn. Sci. 11, 126-135.

McCarthy, G., Puce, A., Gore, J. C., and Allison, T. (1997). Face-specific processing in the human fuisiform gyrus. J. Cogn. Neurosci. 9, 605-610.

McHugh, T. J., Jones, M. W., Quinn, J. J., Balthasar, N., Coppari, R., Elmquist, J. K., Lowell, B. B., Fanselow, M. S., Wilson, M. A., and Tonegawa, S. (2007). Dentate gyrus NMDA receptors mediate rapid pattern separation in the hippocampal network. Science 317, 94-99.

McNaughton, B. L., Battaglia, F. P., Jensen, O., Moser, E. I., and Moser, M.-B. (2006). Path integration and the neural basis of the "cognitive map." Nat. Rev. Neurosci. 7 , 663-678.

McTighe, S. M., Cowell, R. A., Winters, B. D., Bussey, T. J., and Saksida, L. M. (2010). Paradoxical false memory for objects after brain damage. Science 330, 1408-1410.

McTighe, S. M., Mar, A. C., Romberg, C., Bussey, T. J., and Saksida, L. M. (2009). A new touchscreen test of pattern separation: effect of hippocampal lesions. Neuroreport 20, 881-885.

Montaldi, D., and Mayes, A. R. (2010). The role of recollection and familiarity in the functional differentiation of the medial temporal lobes. Hippocampus 20, 1291-1314.

Morgan, L. K., Macevoy, S. P., Aguirre, G. K., and Epstein, R. A. (2011). Distances between real-world locations are represented in the human hippocampus. J. Neurosci. 31, 1238-1245.

Morris, R. G., Garrud, P., Rawlins, J. N., and O'Keefe, J. (1982). Place navigation impaired in rats with hippocampal lesions. Nature 297, 681-683.

Morris, R. G., Schenk, F., Tweedie, F., and Jarrard, L. E. (1990). Ibotenate lesions of hippocampus and/or subiculum: dissociating components of allocentric spatial learning. Eur. J. Neurosci. 2, 1016-1028.

Moser, E. I., Kropff, E., and Moser, M. B. (2008). Place cells, grid cells, and the brain's spatial representation system. Annu. Rev. Neurosci. 31, 69-89.

Moses, S. N., and Ryan, J. D. (2006). A comparison and evaluation of the predictions of relational and conjunctive accounts of hippocampal function. Hippocampus 16, 43-65.

Mumby, D. G., Wood, E. R., Duva, C. A., Kornecook, T. J., Pinel, J. P., and Phillips, A. G. (1996). Ischemiainduced object-recognition deficits in rats are attenuated by hippocampal ablation before or soon after ischemia. Behav. Neurosci. 110, 266-281.

Murray, E. A., Baxter, M. G., and Gaffan, D. (1998). Monkeys with rhinal cortex damage or neurotoxic hippocampal lesions are impaired on spatial scene learning and object reversals. Behav. Neurosci. 112, 1291-12303.

Murray, E. A., and Bussey, T. J. (1999). Perceptual-mnemonic functions of the perirhinal cortex. Trends $\operatorname{Cog} n$. Sci. 3, 142-151.

Murray, E. A., Bussey, T. J., and Saksida, L. M. (2007). Visual perception and memory: a new view of medial temporal lobe function in primates and rodents. Annu. Rev. Neurosci. 30, 99-122.

Murray, E. A., and Mishkin, M. (1998). Object recognition and location memory in monkeys with excitotoxic lesions of the amygdala and hippocampus. J. Neurosci. 18, 6568-6582.

Nichols, E. A., Kao, Y. C., Verfaellie, M., and Gabrieli, J. D. (2006). Working memory and long-term memory for faces: evidence from fMRI and global amnesia for involvement of the medial temporal lobes. Hippocampus 16, 604-616.

Norman, K. A., and O'Reilly, R. C. (2003). Modeling hippocampal and neocortical contributions to recognition memory: a complementarylearning-systems approach. Psychol. Rev. 110, 611-646.

O'Keefe, J. (1976). Place units in the hippocampus of the freely moving rat. Exp. Neurol. 51, 78-109.

O'Keefe, J., and Burgess, N. (2005). Dual phase and rate coding in hippocampal place cells: theoretical significance and relationship to entorhinal grid cells. Hippocampus $15,853-866$.

O’Keefe, J., Burgess, N., Donnett, J. G., Jeffery, K. J., and Maguire, E. A. (1998). Place cells, navigational accuracy, and the human hippocampus. Philos. Trans. R. Soc. Lond. B Biol. Sci. 353, 1333-1340.

O'Keefe, J., and Dostrovsky, J. (1971). The hippocampus as a spatial map. Preliminary evidence from unit activity in the freely-moving rat. Brain Res. 34, 171-175.

O'Keefe, J., and Nadel, L. (1978). The hippocampus as a Cognitive Map. Oxford: Clarendon Press.

O'Keefe, J., and Recce, M. L. (1993). Phase relationship between hippocampal place units and the EEG theta rhythm. Hippocampus 3, 317-330.

Olson, I. R., Moore, K. S., Stark, M., and Chatterjee, A. (2006a). Visual working memory is impaired when the medial temporal lobe is damaged. J. Cogn. Neurosci. 18, 1087-1097.

Olson, I. R., Page, K., Moore, K. S., Chatterjee, A., and Verfaellie, M. (2006b). Working memory for conjunctions relies on the medial temporal lobe. J. Neurosci. 26, 4596-4601.

O'Neil, E. B., Cate, A. D., and Kohler, S. (2009). Perirhinal cortex contributes to accuracy in recognition memory and perceptual discriminations. J. Neurosci. 29, 8329-8334.

O'Neil, E. B., Protzner, A. B., McCormick, C., McLean, D. A., Poppenk, J., Cate, A. D., and Kohler, S. (2012). Distinct patterns of functional and effective connectivity between perirhinal cortex and other cortical regions in recognition memory and perceptual discrimination. Cereb. Cortex. 22, 74-85.

Ono, T., Nakamura, K., Fukuda, M., and Tamura, R. (1991). Place recognition responses of neurons in monkey hippocampus. Neurosci. Lett. 121, 194-198.

Ono, T., Nakamura, K., Nishijo, H., and Eifuku, S. (1993). Monkey hippocampal neurons related to spatial and nonspatial functions. $J$. Neurophysiol. 70, 1516-1529.

O'Reilly, R. C., and McClelland, J. L. (1994). Hippocampal conjunctive encoding, storage, and recall: avoiding a trade-off. Hippocampus 4, 661-682.

Qin, S., Piekema, C., Petersson, K. M., Han, B., Luo, J., and Fernandez, G. (2007). Probing the transformation of discontinuous associations into episodic memory: an eventrelated fMRI study. Neuroimage 38, 212-222.

Ranganath, C. (2010). A unified framework for the functional organization of the medial temporal lobes and the phenomenology of episodic memory. Hippocampus 20, 1263-1290

Ranganath, C., and D'Esposito, M. (2001). Medial temporal lobe activity associated with active maintenance of novel information. Neuron $31,865-873$

Rawlins, J. N. (1985). Associations across time: the hippocampus as a temporary memory store. Behav. Brain Sci. 8, 479-496.

Rawlins, J. N., Lyford, G. L., Seferiades, A., Deacon, R. M., and Cassaday, H. J. (1993). Critical determinants of nonspatial working memory deficits in rats with conventional lesions of the hippocampus or fornix. Behav. Neurosci. 107, 420-433.

Riesenhuber, M., and Poggio, T. (1999). Hierarchical models of object recognition in cortex. Nat. Neurosci. 2, 1019-1025.

Robertson, R. G., Rolls, E. T., and Georges-Fran Ois, P. (1998). Spatial view cells in the primate hippocampus: effects of removal of view details. J. Neurophysiol. 79, 1145-1156.

Rolls, E. T. (1999). Spatial view cells and the representation of place in the primate hippocampus. Hippocampus 9, 467-480.

Rolls, E. T., Robertson, R. G., and Georges-Francois, P. (1997). Spatial view cells in the primate hippocampus. Eur. J. Neurosci. 9, 1789-1794.

Rolls, E. T., Stringer, S. M., and Elliot, T. (2006). Entorhinal cortex grid cells can map to hippocampal place cells by competitive learning. Network 17, 447-465. 
Rosenbaum, R. S., Köhler, S., Schacter, D. L., Moscovitch, M., Westmacott, R., Black, S. E., Gao, F., and Tulving, E. (2005). The case of K.C.: contributions of a memoryimpaired person to memory theory. Neuropsychologia 43, 989-1021.

Rosenbaum, R. S., Priselac, S., Kohler, S., Black, S. E., Gao, F., Nadel, L., and Moscovitch, M. (2000). Remote spatial memory in an amnesic person with extensive bilateral hippocampal lesions. Nat. Neurosci. 3, 1044-1048.

Ryan, J. D., and Cohen, N. J. (2004). Processing and short-term retention of relational information in amnesia. Neuropsychologia 42, 497-511.

Ryan, L., Lin, C. Y., Ketcham, K., and Nadel, L. (2010). The role of medial temporal lobe in retrieving spatial and nonspatial relations from episodic and semantic memory. Hippocampus 20, 11-18.

Saksida, L. M., and Bussey, T. J. (2010). The representational-hierarchical view of amnesia: translation from animal to human. Neuropsychologia 48, 2370-2384.

Scoville, W. B., and Milner, B. (1957). Loss of recent memory after bilateral hippocampal lesions. $J$. Neurochem. 20, 11-21.

Shrager, Y., Gold, J. J., Hopkins, R. O., and Squire, L. R. (2006). Intact visual perception in memoryimpaired patients with medial temporal lobe lesions. J. Neurosci. 26, 2235-2240.

Shrager, Y., Levy, D. A., Hopkins, R. O., and Squire, L. R. (2008). Working memory and the organization of brain systems. J. Neurosci. 28 , 4818-4822.

Spiers, H. J., Burgess, N., Hartley, T., Vargha-Khadem, F., and O'Keefe, J. (2001). Bilateral hippocampal pathology impairs topographical and episodic memory but not visual pattern matching. Hippocampus 11, 715-725.

Spiers, H. J., and Maguire, E. A. (2006). Thoughts, behaviour, and brain dynamics during navigation in the real world. Neuroimage 31, 1826-1840.

Spiers, H. J., and Maguire, E. A. (2007). A navigational guidance system in the human brain. Hippocampus 17, 618-626.

Squire, L., and Zola-Morgan, S. (1991). The medial temporal lobe memory system. Science 253, 1380-1386.

Squire, L. R., Stark, C. E., and Clark, R. E. (2004). The medial temporal lobe. Annu. Rev. Neurosci. 27, 279-306.

Squire, L. R., and Wixted, J. T. (2011). The cognitive neuroscience of human memory since H.M. Annu. Rev. Neurosci. 34, 259-288.

Staresina, B. P., and Davachi, L. (2009). Mind the gap: binding experiences across space and time in the human hippocampus. Neuron 63, 267-276.

Stark, C. E., and Squire, L. R. (2000). Intact visual perceptual discrimination in humans in the absence of perirhinal cortex. Learn. Mem. 7, 273-278.

Stern, C. E., Sherman, S. J., Kirchhoff, B. A., and Hasselmo, M. E. (2001). Medial temporal and prefrontal contributions to working memory tasks with novel and familiar stimuli. Hippocampus 11, 337-346.

Suzuki, W. A. (2009). Perception and the medial temporal lobe: evaluating the current evidence. Neuron 61 , 657-666.

Suzuki, W. A., and Baxter, M. G. (2009). Memory, perception, and the medial temporal lobe: a synthesis of opinions. Neuron 61, 678-679.

Talpos, J. C., McTighe, S. M., Dias, R., Saksida, L. M., and Bussey, T. J. (2010). Trial-unique, delayed nonmatching-to-location (TUNL): a novel, highly hippocampusdependent automated touchscreen test of location memory and pattern separation. Neurobiol. Learn. Mem. $94,341-352$.

Taylor, K. J., Henson, R. N., and Graham, K. S. (2007). Recognition memory for faces and scenes in amnesia: dissociable roles of medial temporal lobe structures. Neuropsychologia 45, 2428-2438.

Tolman, E. C. (1948). Cognitive maps in rats and men. Psychol. Rev. 55, 189-208.

Treves, A., and Rolls, E. T. (1994). Computational analysis of the role of the hippocampus in memory. Hippocampus 4, 374-391.

Tubridy, S., and Davachi, L. (2011) Medial temporal lobe contributions to episodic sequence encoding. Cereb. Cortex 21, 272-280.

Ungerleider, L., and Mishkin, M. (1982). "Two cortical visual systems," in Analysis of Visual Behavior, eds D. Ingle, M. Goodale and R. Mansfield. (Boston: MIT press), 549-586.

Vann, S. D., Tsivilis, D., Denby, C. E., Quamme, J. R., Yonelinas, A P., Aggleton, J. P., Montaldi, D., and Mayes, A. R. (2009). Impaired recollection but spared familiarity in patients with extended hippocampal system damage revealed by 3 convergent methods. Proc. Natl. Acad. Sci. U.S.A. 106 5442-5447.

Wallenstein, G. V., Eichenbaum, H., and Hasselmo, M. E. (1998). The hippocampus as an associator of discontiguous events. Trends Neurosci. 21, 317-323.

Warren, D. E., Duff, M. C., Tranel, D. and Cohen, N. J. (2010). Media temporal lobe damage impairs representation of simple stimuli. Front. Hum. Neurosci. 4:35. doi: 10.3389/fnhum.2010.00035
Wood, E. R., Dudchenko, P. A. Robitsek, R. J., and Eichenbaum, H. (2000). Hippocampal neurons encode information about different types of memory episodes occurring in the same location. Neuron 27, 623-633.

Yassa, M. A., and Stark, C. E. (2011). Pattern separation in the hippocampus. Trends Neurosci. 34, 515-525.

Yonelinas, A. P., Aly, M., Wang, W. C., and Koen, J. D. (2010). Recollection and familiarity: examining controversial assumptions and new directions. Hippocampus 20, 1178-1194.

Yonelinas, A. P., Kroll, N. E., Quamme, J. R., Lazzara, M. M., Sauve, M. J., Widaman, K. F., and Knight, R. T. (2002). Effects of extensive temporal lobe damage or mild hypoxia on recollection and familiarity. Nat Neurosci. 5, 1236-1241.

Conflict of Interest Statement: The authors declare that the research was conducted in the absence of any commercial or financial relationships that could be construed as a potential conflict of interest.

Received: 16 October 2011; accepted: 30 March 2012; published online: 17 April 2012.

Citation: Lee ACH, Yeung $L-K$ and Barense MD (2012) The hippocampus and visual perception. Front. Hum. Neurosci. 6:91. doi: 10.3389/fnhum 2012.00091

Copyright (๑) 2012 Lee, Yeung and Barense. This is an open-access article distributed under the terms of the Creative Commons Attribution Non Commercial License, which permits non-commercial use, distribution, and reproduction in other forums, provided the original authors and source are credited. 